\title{
Neutrino $\mu-\tau$ reflection symmetry and its breaking in the minimal seesaw
}

\author{
Zhi-Cheng Liu, Chong-Xing Yue and Zhen-Hua Zhao \\ Department of Physics, Liaoning Normal University, \\ No. 850 Huanghe Road, Dalian 116029, China \\ E-mail: zhzhao@itp.ac.cn
}

ABSTRACT: In this paper, we attempt to implement the neutrino $\mu-\tau$ reflection symmetry (which predicts $\theta_{23}=\pi / 4$ and $\delta= \pm \pi / 2$ as well as trivial Majorana phases) in the minimal seesaw (which enables us to fix the neutrino masses). For some direct (the preliminary experimental hints towards $\theta_{23} \neq \pi / 4$ and $\delta \neq-\pi / 2$ ) and indirect (inclusion of the renormalization group equation effect and implementation of the leptogenesis mechanism) reasons, we particularly study the breakings of this symmetry and their phenomenological consequences.

Keywords: Discrete Symmetries, Neutrino Physics, CP violation

ArXiv EPrint: 1707.05535 


\section{Contents}

1 Introduction 1

$2 \quad \mu-\tau$ reflection symmetry in the minimal seesaw 3

3 Breaking of the $\mu-\tau$ reflection symmetry $\quad 7$

4 RGE triggered symmetry breaking $\quad 10$

5 Leptogenesis and the symmetry breaking $\quad 12$

$\begin{array}{lll}6 & \text { Summary } & 16\end{array}$

\section{Introduction}

As is known, the discovery of neutrino oscillations indicates that neutrinos are massive and mixed [1]. On the one hand, the most popular way of generating neutrino masses is to invoke the seesaw mechanism which allows for a natural explanation of their smallness. In the type-I seesaw mechanism [2-6], one usually introduces three right-handed neutrino fields $N_{i}$ (for $i=1,2,3$ ). They not only take part in Yukawa interactions with the lefthanded neutrino fields which lead to a Dirac mass matrix $M_{\mathrm{D}}$, but also have a Majorana mass matrix $M_{\mathrm{N}}$ of themselves. Under the assumption of $M_{\mathrm{N}} \gg M_{\mathrm{D}}$, the effective mass matrix for light neutrinos is obtained as $[2-6]$

$$
M_{\nu}=-M_{\mathrm{D}} M_{\mathrm{N}}^{-1} M_{\mathrm{D}}^{\mathrm{T}} .
$$

The overall minus sign here is of no physical meaning and will be neglected in the following discussions. On the other hand, the neutrino mixing arises from a mismatch between their mass and flavor eigenstates and is described by a $3 \times 3$ unitary matrix $U=U_{l}^{\dagger} U_{\nu}[7,8]$ with $U_{l}$ and $U_{\nu}$ being respectively the unitary matrix for diagonalizing the charged-lepton mass matrix $M_{l}$ and $M_{\nu}$. In the standard parametrization, $U$ reads [1]

$$
U \equiv P_{\phi} V P_{\nu}=P_{\phi}\left(\begin{array}{ccc}
c_{12} c_{13} & s_{12} c_{13} & s_{13} e^{-\mathrm{i} \delta} \\
-s_{12} c_{23}-c_{12} s_{23} s_{13} e^{\mathrm{i} \delta} & c_{12} c_{23}-s_{12} s_{23} s_{13} e^{\mathrm{i} \delta} & s_{23} c_{13} \\
s_{12} s_{23}-c_{12} c_{23} s_{13} e^{\mathrm{i} \delta} & -c_{12} s_{23}-s_{12} c_{23} s_{13} e^{\mathrm{i} \delta} & c_{23} c_{13}
\end{array}\right) P_{\nu} .
$$

Here $P_{\phi}=\operatorname{Diag}\left(e^{\mathrm{i} \phi_{1}}, e^{\mathrm{i} \phi_{2}}, e^{\mathrm{i} \phi_{3}}\right)$ consists of three unphysical phases that can be removed via the rephasing of charged-lepton fields, while $P_{\nu}=\operatorname{Diag}\left(e^{\mathrm{i} \rho}, e^{\mathrm{i} \sigma}, 1\right)$ contains two Majorana phases. Similar to the CKM matrix, $V$ possesses a Dirac phase $\delta$ and three mixing angles $\theta_{i j}($ for $i j=12,13,23)$. For convenience, the abbreviations $c_{i j}=\cos \theta_{i j}$ and $s_{i j}=\sin \theta_{i j}$ 
have been used. Besides the mixing parameters, neutrino oscillations are also regulated by two neutrino mass-squared differences $\Delta m_{i j}^{2}=m_{i}^{2}-m_{j}^{2}$ (for $i j=21,31$ ) [9]

$$
\Delta m_{21}^{2}=(7.50 \pm 0.18) \times 10^{-5} \mathrm{eV}^{2}, \quad\left|\Delta m_{31}^{2}\right|=(2.524 \pm 0.039) \times 10^{-3} \mathrm{eV}^{2} .
$$

In comparison, the absolute neutrino mass scale has to be inferred from non-oscillatory experiments, ${ }^{1}$ and a definite result is still missing. Note that the sign of $\Delta m_{31}^{2}$ has not yet been determined, thereby allowing for two possible mass orderings $m_{1}<m_{2}<m_{3}$ (the normal hierarchy, NH for short) and $m_{3}<m_{1}<m_{2}$ (the inverted hierarchy, IH for short). It turns out that the fitted values of mixing parameters depend on the mass ordering in a certain way: $\theta_{13}, \theta_{23}$ and $\delta$ take the values

$$
\sin ^{2} \theta_{13}=0.02166 \pm 0.00075, \quad \sin ^{2} \theta_{23}=0.441 \pm 0.024, \quad \delta=261^{\circ} \pm 55^{\circ},
$$

in the $\mathrm{NH}$ case, or

$$
\sin ^{2} \theta_{13}=0.02179 \pm 0.00076, \quad \sin ^{2} \theta_{23}=0.587 \pm 0.022, \quad \delta=277^{\circ} \pm 43^{\circ},
$$

in the IH case, whereas $\theta_{12}$ takes the value $\sin ^{2} \theta_{12}=0.306 \pm 0.012$ in either case [9].

How to understand the observed neutrino mixing poses an interesting question. As one can see, the measured $\theta_{12}, \theta_{23}$ and $\delta$ are close to some special values

$$
\sin ^{2} \theta_{12}=\frac{1}{3}, \quad \sin ^{2} \theta_{23}=\frac{1}{2}, \quad \delta=-\frac{\pi}{2} .
$$

These remarkable coincidences invite us to speculate that some flavor symmetry has played an important role in shaping the neutrino mixing. ${ }^{2}$ In this connection, the $\mu-\tau$ reflection symmetry [14] serves as a unique candidate: in the basis of $M_{l}$ being diagonal, $M_{\nu}$ should stay invariant with respect to the transformations ${ }^{3}$

$$
\nu_{e} \leftrightarrow \nu_{e}^{c}, \quad \nu_{\mu} \leftrightarrow \nu_{\tau}^{c}, \quad \nu_{\tau} \leftrightarrow \nu_{\mu}^{c},
$$

and have its elements $M_{\alpha \beta}$ (for $\left.\alpha, \beta=e, \mu, \tau\right)$ obeying the conditions

$$
M_{e \mu}=M_{e \tau}^{*}, \quad M_{\mu \mu}=M_{\tau \tau}^{*}, \quad M_{e e} \text { and } M_{\mu \tau} \text { being real . }
$$

Consequently, $U_{l}$ is a diagonal phase matrix of no physical meaning $U_{l}=$ $\operatorname{Diag}\left(e^{\mathrm{i} \varphi_{1}}, e^{\mathrm{i} \varphi_{2}}, e^{\mathrm{i} \varphi_{3}}\right)$ where $\varphi_{1,2,3}$ can take arbitrary values. On the other hand, as a result of the six real conditions for $M_{\nu}$ given by eq. (1.8), there are six predictions [17]

$$
\phi_{1}=0, \quad \phi_{2}=-\phi_{3}, \quad \theta_{23}=\pi / 4, \quad \delta= \pm \pi / 2, \quad \rho, \sigma=0 \text { or } \pi / 2,
$$

for the mixing parameters of $U_{\nu}$ which itself takes a form as given by eq. (1.2). Furthermore, unlike the $\mu-\tau$ interchange symmetry $[18-21]^{4}$ which predicts $\theta_{13}=0$, the $\mu-\tau$ reflection

\footnotetext{
${ }^{1}$ See e.g. refs. [10, 11].

${ }^{2}$ For a review with extensive references see refs. [12, 13].

${ }^{3}$ This operation is a combination of the $\mu-\tau$ exchange and CP conjugate transformations - a specific example of the generalized CP transformations [15, 16].

${ }^{4}$ For a review with extensive references see ref. [22].
} 
symmetry allows for an arbitrary $\theta_{13}$. Thanks to these interesting consequences, this symmetry has been attracting a lot of attention recently. ${ }^{5}$

Nevertheless, the neutrino masses receive no clues from the $\mu-\tau$ reflection symmetry. But they will be fixed if the smallest one $\left(m_{1}\right.$ in the $\mathrm{NH}$ case, $m_{3}$ in the IH case) is to vanish, a possibility that is experimentally allowed and can be accommodated by the minimal seesaw ${ }^{6}$ where only two right-handed neutrino fields $N_{1,2}$ are involved. In this paper, we just attempt to combine the $\mu-\tau$ reflection symmetry with the minimal seesaw so that both the neutrino mixing and mass spectrum can be settled. The implications of such a marriage are discussed in the next section. In consideration of the preliminary experimental results towards $\theta_{23} \neq \pi / 4^{7}$ and $\delta \neq-\pi / 2$, we particularly explore the possible symmetry breakings and their impacts on the mixing parameters in section 3 . Section 4 is devoted to the specific symmetry breaking triggered by the renormalization group equation (RGE) effect. In section 5, the operation of leptogenesis in this framework will be studied. Finally, our main results are summarized in section 6 .

\section{$2 \mu-\tau$ reflection symmetry in the minimal seesaw}

Conversely, one can reconstruct an $M_{\nu}$ of the $\mu-\tau$ reflection symmetry in terms of the $U_{\nu}$ characterized by eq. (1.9) and neutrino masses by means of the relation $M_{\nu}=$ $U_{\nu} \operatorname{Diag}\left(m_{1}, m_{2}, m_{3}\right) U_{\nu}^{\mathrm{T}}$. In the situation of one neutrino mass being vanishing, the reconstructed $M_{\nu}$ in such a way will take a form as

$$
\begin{aligned}
M_{e e} & =\bar{m}_{2} s_{12}^{2} c_{13}^{2}-m_{3} s_{13}^{2}, \\
M_{e \mu} & =\frac{1}{\sqrt{2}}\left[\bar{m}_{2} s_{12}\left(c_{12}+\mathrm{i} s_{12} \bar{s}_{13}\right)+\mathrm{i} m_{3} \bar{s}_{13}\right] c_{13} e^{\mathrm{i} \phi}, \\
M_{\mu \mu} & =\frac{1}{2}\left[\bar{m}_{2}\left(c_{12}+\mathrm{i} s_{12} \bar{s}_{13}\right)^{2}+m_{3} c_{13}^{2}\right] e^{2 \mathrm{i} \phi}, \\
M_{\mu \tau} & =\frac{1}{2}\left[\bar{m}_{2}\left(c_{12}^{2}+s_{12}^{2} s_{13}^{2}\right)-m_{3} c_{13}^{2}\right],
\end{aligned}
$$

in the $\mathrm{NH}$ case, or

$$
\begin{aligned}
M_{e e} & =\left[m_{1} c_{12}^{2}+\bar{m}_{2} s_{12}^{2}\right] c_{13}^{2}, \\
M_{e \mu} & =\frac{1}{\sqrt{2}}\left[m_{1} c_{12}\left(-s_{12}+\mathrm{i} c_{12} \bar{s}_{13}\right)+\bar{m}_{2} s_{12}\left(c_{12}+\mathrm{i} s_{12} \bar{s}_{13}\right)\right] c_{13} e^{\mathrm{i} \phi}, \\
M_{\mu \mu} & =\frac{1}{2}\left[m_{1}\left(s_{12}-\mathrm{i} c_{12} \bar{s}_{13}\right)^{2}+\bar{m}_{2}\left(c_{12}+\mathrm{i} s_{12} \bar{s}_{13}\right)^{2}\right] e^{2 \mathrm{i} \phi}, \\
M_{\mu \tau} & =\frac{1}{2}\left[m_{1}\left(s_{12}^{2}+c_{12}^{2} s_{13}^{2}\right)+\bar{m}_{2}\left(c_{12}^{2}+s_{12}^{2} s_{13}^{2}\right)\right],
\end{aligned}
$$

in the IH case, where $\bar{m}_{2} \equiv m_{2} e^{2 \mathrm{i} \sigma}$ and $\bar{s}_{13} \equiv-\mathrm{i} s_{13} e^{\mathrm{i} \delta}$ have been defined. Note that $M_{e \mu}$ and $M_{\mu \mu}$ depend on the unphysical phase $\phi \equiv \phi_{2}=-\phi_{3}$ whose value can be chosen freely

\footnotetext{
${ }^{5}$ For an incomplete list see refs. [23-40].

${ }^{6}$ For an incomplete list see refs. [41-61].

${ }^{7}$ In particular, a recent result reported by the NOvA collaboration $\left(\theta_{23}=39.5^{\circ} \pm 1.7^{\circ}\right.$ or $52.1^{\circ} \pm 1.7^{\circ}$ in the $\mathrm{NH}$ case) disfavors the possibility of $\theta_{23}=45^{\circ}$ at a $2.6 \sigma$ level [62].
} 


\begin{tabular}{|c|c|c|c|c|}
\hline${ }_{[\delta, \sigma]} M_{\alpha \beta}(\mathrm{eV})$ & $M_{e e}\left(\times 10^{-2}\right)$ & $M_{e \mu}\left(\times 10^{-2} e^{\mathrm{i} \phi}\right)$ & $M_{\mu \mu}\left(\times 10^{-2} e^{2 \mathrm{i} \phi}\right)$ & $M_{\mu \tau}\left(\times 10^{-2}\right)$ \\
\hline$[\pi / 2,0]$ & 0.15 & $0.28+0.54 \mathrm{i}$ & $2.76+0.06 \mathrm{i}$ & -2.15 \\
\hline$[-\pi / 2,0]$ & 0.15 & $0.28-0.54 \mathrm{i}$ & $2.76-0.06 \mathrm{i}$ & -2.15 \\
\hline$[\pi / 2, \pi / 2]$ & -0.37 & $-0.28+0.49 \mathrm{i}$ & $2.16-0.06 \mathrm{i}$ & -2.76 \\
\hline$[-\pi / 2, \pi / 2]$ & -0.37 & $-0.28-0.49 \mathrm{i}$ & $2.16+0.06 \mathrm{i}$ & -2.76 \\
\hline
\end{tabular}

Table 1. The possible values of $M_{\alpha \beta}$ for various combinations of $\delta$ and $\sigma$ in the NH case.

\begin{tabular}{|c|c|c|c|c|}
\hline$M_{[\delta, \sigma]}(\mathrm{eV})$ & $M_{e e}\left(\times 10^{-2}\right)$ & $M_{e \mu}\left(\times 10^{-2} e^{\mathrm{i} \phi}\right)$ & $M_{\mu \mu}\left(\times 10^{-2} e^{2 \mathrm{i} \phi}\right)$ & $M_{\mu \tau}\left(\times 10^{-2}\right)$ \\
\hline$[\pi / 2,0]$ & 4.86 & $0.02+0.51 \mathrm{i}$ & $2.45+0.01 \mathrm{i}$ & 2.56 \\
\hline$[-\pi / 2,0]$ & 4.86 & $0.02-0.51 \mathrm{i}$ & $2.45-0.01 \mathrm{i}$ & 2.56 \\
\hline$[\pi / 2, \pi / 2]$ & 1.86 & $-3.21+0.20 \mathrm{i}$ & $-1.01-0.68 \mathrm{i}$ & -0.97 \\
\hline$[-\pi / 2, \pi / 2]$ & 1.86 & $-3.21-0.20 \mathrm{i}$ & $-1.01+0.68 \mathrm{i}$ & -0.97 \\
\hline
\end{tabular}

Table 2. The possible values of $M_{\alpha \beta}$ for various combinations of $\delta$ and $\sigma$ in the IH case.

without affecting the physical results. And there is only one effective Majorana phase which we assign for $m_{2}$ (i.e., $\sigma$ ). Because of the condition $\operatorname{Det}\left(M_{\nu}\right)=0,{ }^{8}$ only five out of the six real components of these elements are independent. Taking the best-fit values for $\theta_{13}, \theta_{12}$, $\Delta m_{21}^{2}$ and $\left|\Delta m_{31}^{2}\right|$ as input, we give the values of $M_{\alpha \beta}$ for various combinations of $\delta$ and $\sigma$ (i.e., $[\delta, \sigma]=[\pi / 2,0],[-\pi / 2,0],[\pi / 2, \pi / 2]$ and $[-\pi / 2, \pi / 2]$ ) in the $\mathrm{NH}$ (table 1 ) and IH (table 2) cases. As is well known, the size of $\left|M_{e e}\right|$ which governs the rate of neutrino-less double beta decays ${ }^{9}$ is much larger in the $\mathrm{IH}$ case than in the $\mathrm{NH}$ case. In the NH case, the elements exhibit a hierarchical structure as $\left|M_{\mu \mu}\right| \sim\left|M_{\mu \tau}\right| \gg\left|M_{e e}\right| \sim\left|M_{e \mu}\right|$, implying that they might have received contributions from different levels [67]. In the IH case, $\left|M_{e e}\right|$ (so does $\left|M_{e \mu}\right|$ for $\sigma=0$ ) becomes comparable to $\left|M_{\mu \mu}\right|$ and $\left|M_{\mu \tau}\right|$. But for $\sigma=\pi / 2, M_{e \mu}$ will have a magnitude much smaller than the other ones as a result of the heavy cancellation between its two components respectively associated with $m_{1}$ and $\bar{m}_{2}$. By choosing the value of $\phi$ in such a way that the phase of $M_{e \mu}$ or $M_{\mu \mu}$ cancels out, one may convert $M_{\nu}$ to a simpler form as given by tables $3-4$.

The $M_{\nu}$ given by eqs. (2.1)-(2.2) can be viewed as a result of the minimal seesaw: the Dirac mass matrix coupling $N_{1,2}$ with the left-handed neutrino fields is assumed to appear

\footnotetext{
${ }^{8}$ Since the determinant of an $M_{\nu}$ obeying the $\mu$ - $\tau$ reflection symmetry is always real, this condition only amounts to one constraint.

${ }^{9}$ For a review with extensive references see refs. [63-66].
} 


\begin{tabular}{|c|c|c|c|c|}
\hline$M_{[\delta, \sigma]} M_{\alpha \beta}(\mathrm{eV})$ & $M_{e \mu}\left(\times 10^{-2}\right)$ & $M_{\mu \mu}\left(\times 10^{-2}\right)$ & $M_{e \mu}\left(\times 10^{-2}\right)$ & $M_{\mu \mu}\left(\times 10^{-2}\right)$ \\
\hline$[\pi / 2,0]$ & 0.61 & $-1.56-2.27 \mathrm{i}$ & $0.28+0.54 \mathrm{i}$ & 2.76 \\
\hline$[-\pi / 2,0]$ & 0.61 & $-1.56+2.27 \mathrm{i}$ & $0.28-0.54 \mathrm{i}$ & 2.76 \\
\hline$[\pi / 2, \pi / 2]$ & 0.56 & $-1.05+1.89 \mathrm{i}$ & $-0.29+0.49 \mathrm{i}$ & 2.16 \\
\hline$[-\pi / 2, \pi / 2]$ & 0.56 & $-1.05-1.89 \mathrm{i}$ & $-0.29-0.49 \mathrm{i}$ & 2.16 \\
\hline
\end{tabular}

Table 3. The possible values of $M_{e \mu}$ and $M_{\mu \mu}$ for various combinations of $\delta$ and $\sigma$ in the NH case, after one of them is made real by a particular value of $\phi$.

\begin{tabular}{|c|c|c|c|c|}
\hline$M_{\alpha \beta}(\mathrm{eV})$ & $M_{e \mu}\left(\times 10^{-2}\right)$ & $M_{\mu \mu}\left(\times 10^{-2}\right)$ & $M_{e \mu}\left(\times 10^{-2}\right)$ & $M_{\mu \mu}\left(\times 10^{-2}\right)$ \\
\hline$[\pi / 2,0]$ & 0.51 & $-2.43-0.24 \mathrm{i}$ & $0.02+0.51 \mathrm{i}$ & 2.45 \\
\hline$[-\pi / 2,0]$ & 0.51 & $-2.43+0.24 \mathrm{i}$ & $0.02-0.51 \mathrm{i}$ & 2.45 \\
\hline$[\pi / 2, \pi / 2]$ & 3.22 & $-0.92-0.80 \mathrm{i}$ & $-1.13-3.02 \mathrm{i}$ & 1.21 \\
\hline$[-\pi / 2, \pi / 2]$ & 3.22 & $-0.92+0.80 \mathrm{i}$ & $-1.13+3.02 \mathrm{i}$ & 1.21 \\
\hline
\end{tabular}

Table 4. The possible values of $M_{e \mu}$ and $M_{\mu \mu}$ for various combinations of $\delta$ and $\sigma$ in the IH case, after one of them is made real by a particular value of $\phi$.

as $[68]$

$$
M_{\mathrm{D}}=\left(\begin{array}{cc}
a_{1} \sqrt{M_{1}} & \sqrt{M_{2}} b_{1} \\
e^{\mathrm{i} \phi_{a}} a_{2} \sqrt{M_{1}} & \sqrt{M_{2}} b_{2} e^{\mathrm{i} \phi_{b}} \\
e^{-\mathrm{i} \phi_{a}} a_{2} \sqrt{M_{1}} & \sqrt{M_{2}} b_{2} e^{-\mathrm{i} \phi_{b}}
\end{array}\right),
$$

with $a_{1,2}, b_{1,2}, \phi_{a, b}$ and $M_{1,2}$ being real parameters. Apparently, its elements satisfy the conditions of $M_{\mu i}=M_{\tau i}^{*}$ and $M_{e i}$ being real (for $i=1,2$ ). It is easy to see that these conditions still hold when the right-handed neutrino fields experience an orthogonal basis transformation. So, without loss of generality, we choose to work in the basis where the Majorana mass matrix for $N_{1,2}$ is diagonal $M_{\mathrm{N}}=\operatorname{Diag}\left(M_{1}, M_{2}\right)$. By virtue of the seesaw formula in eq. (1.1), we arrive at an effective neutrino mass matrix

$$
M_{\nu}=\left(\begin{array}{ccc}
a_{1}^{2}+b_{1}^{2} & a_{1} a_{2} e^{\mathrm{i} \phi_{a}}+b_{1} b_{2} e^{\mathrm{i} \phi_{b}} & a_{1} a_{2} e^{-\mathrm{i} \phi_{a}}+b_{1} b_{2} e^{-\mathrm{i} \phi_{b}} \\
a_{1} a_{2} e^{\mathrm{i} \phi_{a}}+b_{1} b_{2} e^{\mathrm{i} \phi_{b}} & a_{2}^{2} e^{2 \mathrm{i} \phi_{a}}+b_{2}^{2} e^{2 \mathrm{i} \phi_{b}} & a_{2}^{2}+b_{2}^{2} \\
a_{1} a_{2} e^{-\mathrm{i} \phi_{a}}+b_{1} b_{2} e^{-\mathrm{i} \phi_{b}} & a_{2}^{2}+b_{2}^{2} & a_{2}^{2} e^{-2 \mathrm{i} \phi_{a}}+b_{2}^{2} e^{-2 \mathrm{i} \phi_{b}}
\end{array}\right)
$$


Diagonalizing this $M_{\nu}$ with a $U_{\nu}$ characterized by eq. (1.9) yields the mixing parameters

$$
\begin{aligned}
\tan \theta_{13} & =\frac{a_{2}^{2} \sin \left(2 \phi-2 \phi_{a}\right)+b_{2}^{2} \sin \left(2 \phi-2 \phi_{b}\right)}{-\sqrt{2} \sin \delta\left[a_{1} a_{2} \cos \left(\phi-\phi_{a}\right)+b_{1} b_{2} \cos \left(\phi-\phi_{b}\right)\right]}, \\
\tan 2 \theta_{13} & =\frac{-2 \sqrt{2}\left[a_{1} a_{2} \sin \left(\phi-\phi_{a}\right)+b_{1} b_{2} \sin \left(\phi-\phi_{b}\right)\right]}{\sin \delta\left[a_{1}^{2}+b_{1}^{2}-P\right]} \\
\tan 2 \theta_{12} & =\frac{-2 \sqrt{2} \cos 2 \theta_{13}\left[a_{1} a_{2} \cos \left(\phi-\phi_{a}\right)+b_{1} b_{2} \cos \left(\phi-\phi_{b}\right)\right]}{c_{13}\left[\left(a_{1}^{2}+b_{1}^{2}\right) c_{13}^{2}-P s_{13}^{2}-Q \cos 2 \theta_{13}\right]}
\end{aligned}
$$

and neutrino masses

$$
\begin{aligned}
& \bar{m}_{1}=Q-\frac{\sqrt{2}}{c_{13} t_{12}}\left[a_{1} a_{2} \cos \left(\phi-\phi_{a}\right)+b_{1} b_{2} \cos \left(\phi-\phi_{b}\right)\right], \\
& \bar{m}_{2}=Q+\frac{\sqrt{2} t_{12}}{c_{13}}\left[a_{1} a_{2} \cos \left(\phi-\phi_{a}\right)+b_{1} b_{2} \cos \left(\phi-\phi_{b}\right)\right], \\
& \bar{m}_{3}=\frac{\left(a_{1}^{2}+b_{1}^{2}\right) s_{13}^{2}-P c_{13}^{2}}{\cos 2 \theta_{13}},
\end{aligned}
$$

where $\bar{m}_{1}=m_{1} e^{2 \mathrm{i} \rho}, \bar{m}_{3}=m_{3} e^{2 \mathrm{i} \gamma}($ for $\rho, \gamma=0$ or $\pi / 2)$ and

$$
\begin{aligned}
& P=2 a_{2}^{2} \sin ^{2}\left(\phi-\phi_{a}\right)+2 b_{2}^{2} \sin ^{2}\left(\phi-\phi_{b}\right), \\
& Q=2 a_{2}^{2} \cos ^{2}\left(\phi-\phi_{a}\right)+2 b_{2}^{2} \cos ^{2}\left(\phi-\phi_{b}\right) .
\end{aligned}
$$

For any given values of $a_{1,2}, b_{1,2}$ and $\phi_{a, b}$, one mass will necessarily vanish as promised by the minimal seesaw. ${ }^{10}$ The resulting $\theta_{13}, \phi, \theta_{12}$ and two non-zero masses can be calculated with the help of the other five equations.

If we are to derive the allowed values of $a_{1,2}, b_{1,2}$ and $\phi_{a, b}$ from the measured $\theta_{13}, \theta_{23}$, $\Delta m_{21}^{2}$ and $\left|\Delta m_{31}^{2}\right|$, one just needs to confront the $M_{\nu}$ in eq. (2.4) with the results given by tables 1-2. Above all, it should be noted that both the ee and $\mu \tau$ elements of this $M_{\nu}$ are positive. In order for them to fit in with the corresponding results in tables 1-2, one must have $\sigma=\pi / 2$ (or 0 ) and $M_{\nu} \rightarrow-M_{\nu}$ (or $+M_{\nu}$ ) in the $\mathrm{NH}$ (or IH) case. In light of the unphysical nature of $\phi, \phi_{a, b}-\phi$ rather than $\phi_{a, b}$ will be treated as effective independent parameters. Recall that only five real components of the neutrino mass matrix elements are independent, so the free parameters are more than the constraint equations by one. For this reason, in figure 1 we choose to present the results for $a_{2}, b_{1,2}$ and $\phi_{a, b}-\phi$ as functions of $a_{1}$ (which stands in an equivalent position as $b_{1}$ ). In the numerical calculations here and in the following, the best-fit values for $\theta_{13}, \theta_{12}, \Delta m_{21}^{2}$ and $\left|\Delta m_{31}^{2}\right|$ are input, whereas $\delta$ is specified as $-\pi / 2$. We have only shown the results in the case of both $a_{1}$ and $b_{1}$ being positive. The results in the case of $a_{1}$ or (and) $b_{1}$ being negative can be obtained by simply making the replacement $a_{1} \rightarrow-a_{1}$ combined with $\left(\phi_{a}-\phi\right) \rightarrow\left(\phi_{a}-\phi\right)+\pi$ or (and) $b_{1} \rightarrow-b_{1}$ combined with $\left(\phi_{b}-\phi\right) \rightarrow\left(\phi_{b}-\phi\right)+\pi$. This is because the $M_{\nu}$ in eq. (2.4) keeps invariant under this kind of transformations. It is interesting to find that the possibility of

\footnotetext{
${ }^{10}$ We are left with the difference of the phases associated with two non-zero masses as the effective Majorana phase.
} 


\begin{tabular}{|c|c|c|c|c|c|}
\hline & $b_{1}$ & $\phi_{b}-\phi$ & $a_{2}$ & $b_{2}$ & $\phi_{a}-\phi$ \\
\hline $\mathrm{NH}$ & 0.06 & 1.05 & 0.14 & 0.09 & $1.79,4.93$ \\
\hline $\mathrm{IH}$ & 0.22 & 4.76 & 0.16 & 0.02 & $0, \pi$ \\
\hline
\end{tabular}

Table 5. The possible values of $a_{2}, b_{1,2}$ and $\phi_{a, b}-\phi$ in the particular case of $a_{1}=0$.

$a_{1}=0$ is allowed. The possible values of $a_{2}, b_{1,2}$ and $\phi_{a, b}-\phi$ in such a particular case are listed in table 5. If we further make one of $\phi_{a, b}$ vanish by giving $\phi$ an appropriate value, then we will reach the simplest $M_{\mathrm{D}}$. In the $\mathrm{NH}$ case, for instance, a value of -1.79 or -4.93 for $\phi$ allows us to have $\phi_{a}=0$. (In the meantime, $\phi_{b}$ is fixed to -0.74 or -3.88 .)

\section{Breaking of the $\mu-\tau$ reflection symmetry}

In this section we study the possible breakings of $\mu-\tau$ reflection symmetry and their impacts on the mixing parameters. ${ }^{11}$ The most general perturbation to $M_{\mathrm{D}}$

$$
\delta M_{\mathrm{D}}=\left(\begin{array}{cc}
\delta_{11} \sqrt{M_{1}} & \sqrt{M_{2}} \delta_{12} \\
\delta_{21} \sqrt{M_{1}} & \sqrt{M_{2}} \delta_{22} \\
\delta_{31} \sqrt{M_{1}} & \sqrt{M_{2}} \delta_{32}
\end{array}\right)
$$

can be decomposed into a symmetry-conserving part and a symmetry-violating part:

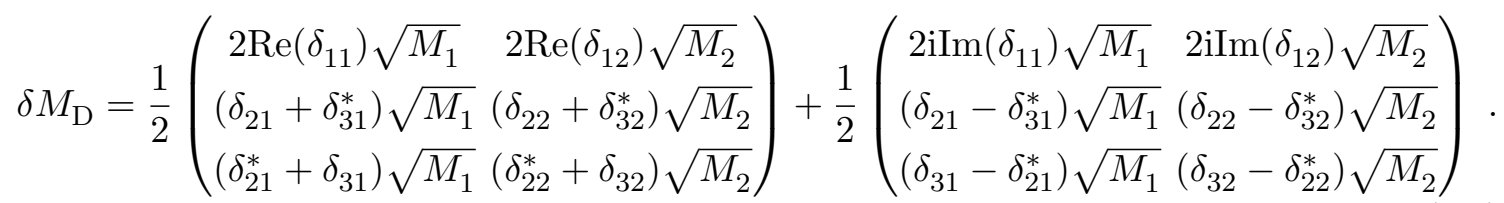

And the full Dirac mass matrix can be reparameterized as

$$
M_{\mathrm{D}}^{\prime}=M_{\mathrm{D}}+\delta M_{\mathrm{D}}=\left(\begin{array}{cc}
\hat{a}_{1}\left(1+\mathrm{i} \epsilon_{1}\right) \sqrt{M_{1}} & \hat{b}_{1}\left(1+\mathrm{i} \epsilon_{2}\right) \sqrt{M_{2}} \\
\hat{a}_{2} e^{\mathrm{i} \hat{\phi}_{a}}\left(1+\epsilon_{3}\right) \sqrt{M_{1}} & \hat{b}_{2} e^{\mathrm{i} \hat{\phi}_{b}}\left(1+\epsilon_{4}\right) \sqrt{M_{2}} \\
\hat{a}_{2} e^{-\mathrm{i} \hat{\phi}_{a}}\left(1-\epsilon_{3}^{*}\right) \sqrt{M_{1}} & \hat{b}_{2} e^{-\mathrm{i} \hat{\phi}_{b}\left(1-\epsilon_{4}^{*}\right) \sqrt{M_{2}}}
\end{array}\right),
$$

with

$$
\begin{array}{ll}
\hat{a}_{1}=a_{1}+\operatorname{Re}\left(\delta_{11}\right), & \hat{a}_{2} e^{\mathrm{i} \hat{\phi}_{a}}=a_{2} e^{\mathrm{i} \phi_{a}}+\frac{\delta_{21}+\delta_{31}^{*}}{2}, \\
\hat{b}_{1}=b_{1}+\operatorname{Re}\left(\delta_{21}\right), & \hat{b}_{2} e^{\mathrm{i} \hat{\phi}_{b}}=b_{2} e^{\mathrm{i} \phi_{b}}+\frac{\delta_{22}+\delta_{32}^{*}}{2},
\end{array}
$$

and

$$
\begin{aligned}
\epsilon_{1} & \equiv \frac{\operatorname{Im}\left(\left(M_{\mathrm{D}}\right)_{e 1}\right)}{\operatorname{Re}\left(\left(M_{\mathrm{D}}\right)_{e 1}\right)}=\frac{\operatorname{Im}\left(\delta_{11}\right)}{a_{1}+\operatorname{Re}\left(\delta_{11}\right)}, \quad \epsilon_{3} \equiv \frac{\left(M_{\mathrm{D}}\right)_{\mu 1}-\left(M_{\mathrm{D}}\right)_{\tau 1}^{*}}{\left(M_{\mathrm{D}}\right)_{\mu 1}+\left(M_{\mathrm{D}}\right)_{\tau 1}^{*}}=\frac{\delta_{21}-\delta_{31}^{*}}{2 a_{2} e^{\mathrm{i} \phi_{a}}+\delta_{21}+\delta_{31}^{*}}, \\
\epsilon_{2} & \equiv \frac{\operatorname{Im}\left(\left(M_{\mathrm{D}}\right)_{e 2}\right)}{\operatorname{Re}\left(\left(M_{\mathrm{D}}\right)_{e 2}\right)}=\frac{\operatorname{Im}\left(\delta_{21}\right)}{a_{1}+\operatorname{Re}\left(\delta_{21}\right)}, \quad \epsilon_{4} \equiv \frac{\left(M_{\mathrm{D}}\right)_{\mu 2}-\left(M_{\mathrm{D}}\right)_{\tau 2}^{*}}{\left(M_{\mathrm{D}}\right)_{\mu 2}+\left(M_{\mathrm{D}}\right)_{\tau 2}^{*}}=\frac{\delta_{22}-\delta_{32}^{*}}{2 b_{2} e^{\mathrm{i} \phi_{b}}+\delta_{22}+\delta_{32}^{*}} .
\end{aligned}
$$

\footnotetext{
${ }^{11}$ For some discussions about the breakings of $\mu-\tau$ interchange symmetry see refs. [69, 70].
} 


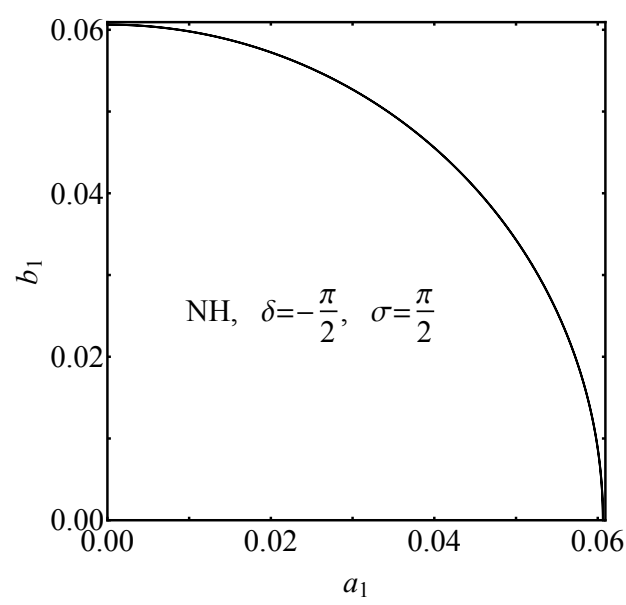

(a)

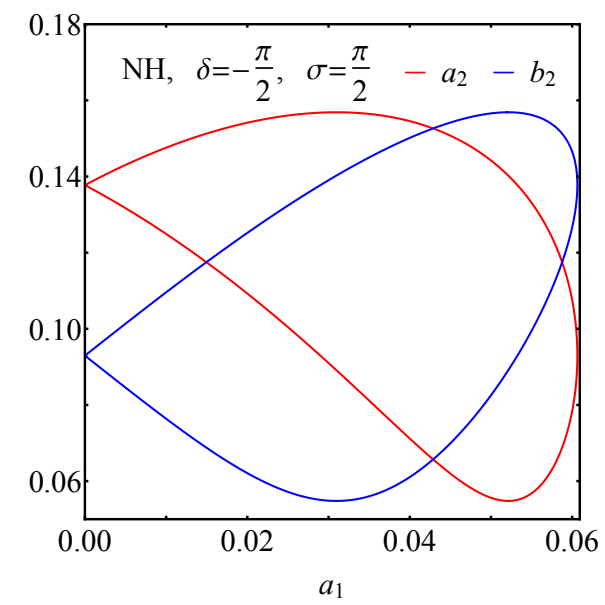

(c)

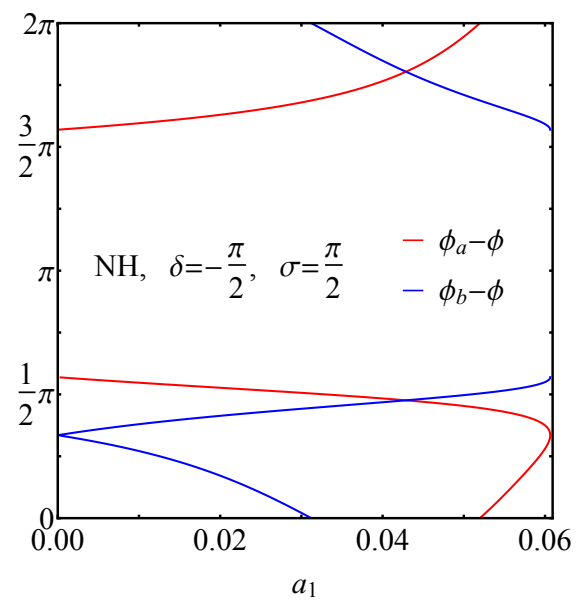

(e)

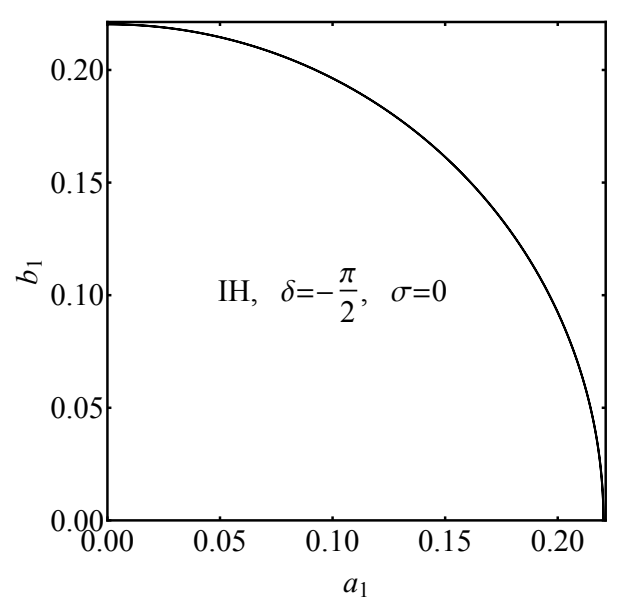

(b)

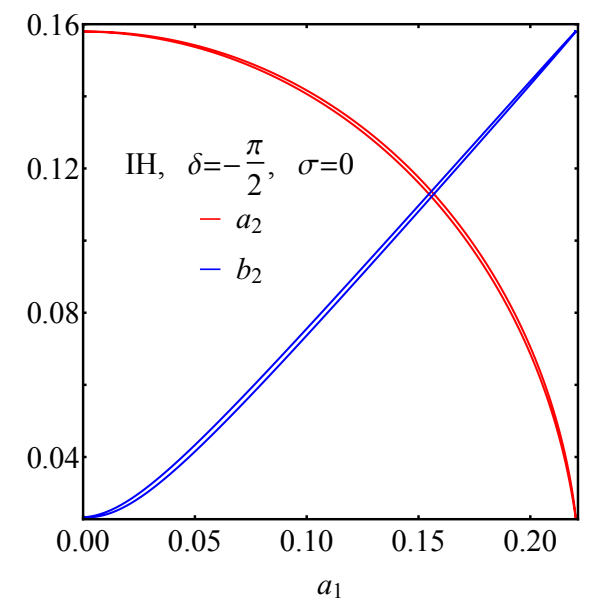

(d)

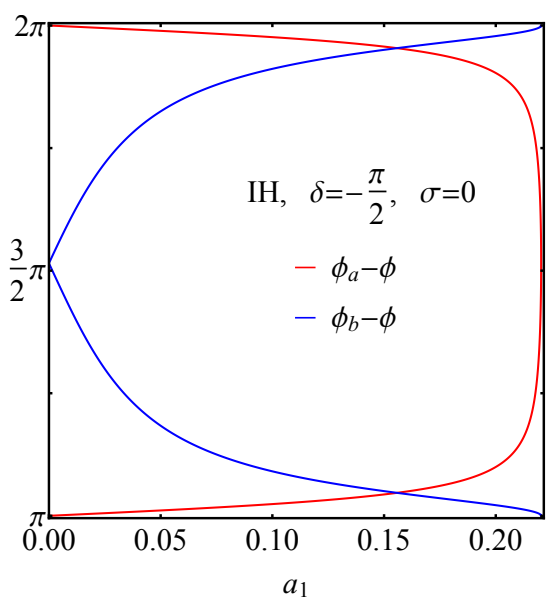

(f)

Figure 1. The allowed values of $a_{2}, b_{1,2}$ and $\phi_{a, b}-\phi$ as functions of $a_{1}$, with $\sigma=\pi / 2$ (or 0 ) in the $\mathrm{NH}$ (or $\mathrm{IH})$ case and $\delta=-\pi / 2$. 
The dimensionless quantities $\epsilon_{1,2,3,4}$ measure the strength of symmetry breaking. They should be small (e.g., $\left|\epsilon_{1,2,3,4}\right| \leq 0.1$ ) in order for $M_{\mathrm{D}}^{\prime}$ to assume an approximate $\mu-\tau$ reflection symmetry.

Since $\epsilon_{2}$ and $\epsilon_{4}$ play equivalent roles as $\epsilon_{1}$ and $\epsilon_{3}$, they will be assumed to vanish in the following discussions. For the sake of simplicity, the hat symbols on $\hat{a}_{1,2}, \hat{b}_{1,2}$ and $\hat{\phi}_{a, b}$ will also be neglected. Consequently, we are led to an effective neutrino mass matrix $M_{\nu}^{\prime}$ of the form

$$
\begin{aligned}
& M_{e e}^{\prime}=a_{1}^{2}\left(1+2 \mathrm{i} \epsilon_{1}\right)+b_{1}^{2} \\
& M_{e \mu}^{\prime}=a_{1} a_{2} e^{\mathrm{i} \phi_{a}}\left(1+\mathrm{i} \epsilon_{1}+\epsilon_{3}\right)+b_{1} b_{2} e^{\mathrm{i} \phi_{b}}, \\
& M_{e \tau}^{\prime}=a_{1} a_{2} e^{-\mathrm{i} \phi_{a}}\left(1+\mathrm{i} \epsilon_{1}-\epsilon_{3}^{*}\right)+b_{1} b_{2} e^{-\mathrm{i} \phi_{b}}, \\
& M_{\mu \tau}^{\prime}=a_{2}^{2}\left[1+2 \mathrm{i} \operatorname{Im}\left(\epsilon_{3}\right)\right]+b_{2}^{2} \\
& M_{\mu \mu}^{\prime}=a_{2}^{2} e^{2 \mathrm{i} \phi_{a}}\left(1+2 \epsilon_{3}\right)+b_{2}^{2} e^{2 \mathrm{i} \phi_{b}} \\
& M_{\tau \tau}^{\prime}=a_{2}^{2} e^{-2 \mathrm{i} \phi_{a}}\left(1-2 \epsilon_{3}^{*}\right)+b_{2}^{2} e^{-2 \mathrm{i} \phi_{b}}
\end{aligned}
$$

at the leading order. The unitary matrix $U_{\nu}^{\prime}$ for diagonalizing $M_{\nu}^{\prime}$ is expected to have some mixing parameters around the special values given by eq. (1.9), with the corresponding deviations

$$
\begin{aligned}
& \Delta \phi_{1}=\phi_{1}^{\prime}-0, \quad \Delta \phi_{2}=\left(\phi_{2}^{\prime}+\phi_{3}^{\prime}\right) / 2-0, \quad \Delta \theta_{23}=\theta_{23}^{\prime}-\pi / 4, \\
& \Delta \delta=\delta^{\prime}-\delta, \quad \Delta \sigma=\sigma^{\prime}-\sigma,
\end{aligned}
$$

being some small quantities. By making series expansions for these mixing-parameter deviations in the diagonalization process, at the leading order we acquire the following relations connecting them with the symmetry-breaking parameters $\epsilon_{1,3}$

$$
\begin{aligned}
& m_{3} s_{13}^{2} \Delta \delta+\bar{m}_{2} s_{12}^{2} \Delta \sigma=\mp\left[a_{1}^{2} \epsilon_{1}-\left(a_{1}^{2}+b_{1}^{2}\right) \Delta \phi_{1}\right] \\
& \sqrt{2}\left[\left(m_{1}-\bar{m}_{2}\right) c_{12} s_{12}+\mathrm{i}\left(m_{1} c_{12}^{2}+\bar{m}_{2} s_{12}^{2}+m_{3}\right) \bar{s}_{13}\right] \Delta \theta_{23} \\
& \quad-\sqrt{2}\left(m_{1} c_{12}^{2}+\bar{m}_{2} s_{12}^{2}-m_{3}\right) \bar{s}_{13} \Delta \delta+2 \sqrt{2} \bar{m}_{2} s_{12}\left(\mathrm{i} c_{12}-s_{12} \bar{s}_{13}\right) \Delta \sigma \\
& =\mp 2\left[a_{1} a_{2} e^{\mathrm{i}\left(\phi_{a}-\phi\right)}\left(\mathrm{i} \epsilon_{1}+\epsilon_{3}-\mathrm{i} \Delta \phi_{1}-\mathrm{i} \Delta \phi_{2}\right)+b_{1} b_{2} e^{\mathrm{i}\left(\phi_{b}-\phi\right)}\left(-\mathrm{i} \Delta \phi_{1}-\mathrm{i} \Delta \phi_{2}\right)\right] \\
& \quad-\left(m_{1} s_{12}^{2}+\bar{m}_{2} c_{12}^{2}-m_{3}\right) \Delta \theta_{23}+\left[\left(m_{1}-\bar{m}_{2}\right) c_{12} s_{12}-\mathrm{i}\left(m_{1} c_{12}^{2}+\bar{m}_{2} s_{12}^{2}\right) \bar{s}_{13}\right] \bar{s}_{13} \Delta \delta \\
& \quad+\bar{m}_{2} c_{12}\left(\mathrm{i} c_{12}-2 s_{12} \bar{s}_{13}\right) \Delta \sigma=\mp 2\left[a_{2}^{2} e^{2 \mathrm{i}\left(\phi_{a}-\phi\right)}\left(\epsilon_{3}-\mathrm{i} \Delta \phi_{2}\right)+b_{2}^{2} e^{2 \mathrm{i}\left(\phi_{b}-\phi\right)}\left(-\mathrm{i} \Delta \phi_{2}\right)\right]
\end{aligned}
$$

where $m_{1}=0$ (or $m_{3}=0$ ) in the $\mathrm{NH}$ (or $\mathrm{IH}$ ) case and the values of $a_{1,2}, b_{1,2}$ and $\phi_{a, b}-\phi$ are the same as those presented in figure 1 . The sign $\mp$ which takes $-($ or + ) in the NH (or $\mathrm{IH})$ case arises from the aforementioned fact that $M_{\nu}$ might need an overall sign change so as to fit in with the numerical results.

By solving these equations in a straightforward way, one will obtain the mixingparameter deviations as some linear functions of $\epsilon_{1}, \operatorname{Re}\left(\epsilon_{3}\right)$ and $\operatorname{Im}\left(\epsilon_{3}\right)$. For illustration, in figure 2 we present the $\Delta \theta_{23}, \Delta \delta$ and $\Delta \sigma$ (as functions of $a_{1}$ ) arising from $\epsilon_{1}=0.1$, $\operatorname{Re}\left(\epsilon_{3}\right)=0.1$ and $\operatorname{Im}\left(\epsilon_{3}\right)=0.1$ in the NH and IH cases. Provided that the linear approximation holds to a good degree (i.e., the expected small quantities are really $\leq \mathcal{O}(0.1)$ ), the 
$\Delta \theta_{23}, \Delta \delta$ and $\Delta \sigma$ generated by other values of $\epsilon_{1}, \operatorname{Re}\left(\epsilon_{3}\right)$ and $\operatorname{Im}\left(\epsilon_{3}\right)$ can be inferred by rescaling these results (according to the linear dependence of mixing-parameter deviations on symmetry-breaking parameters). The results in figure 2 tell us: (a) In the NH case, $\epsilon_{1}=0.1$ may give rise to a $|\Delta \delta|$ as large as 0.1. But the resulting $\left|\Delta \theta_{23}\right|$ and $|\Delta \sigma|$ are desperately small. (b) In the IH case, the $\left|\Delta \theta_{23}\right|,|\Delta \delta|$ and $|\Delta \sigma|$ from $\epsilon_{1}=0.1$ are $\simeq \mathcal{O}(0.01)$. (c) In the NH case, $\operatorname{Re}\left(\epsilon_{3}\right)=0.1$ likely leads to some considerable ( $\simeq 0.1$ or so) $\left|\Delta \theta_{23}\right|,|\Delta \delta|$ and $|\Delta \sigma|$. (d) In the IH case, the $\left|\Delta \theta_{23}\right|$ and $|\Delta \delta|$ induced by $\operatorname{Re}\left(\epsilon_{3}\right)=0.1$ may reach 0.1 and 0.35 (but for distinct values of $a_{1}$ ), while $|\Delta \sigma|$ is rather small. (e)-(f) In both the NH and IH cases, $\operatorname{Im}\left(\epsilon_{3}\right)=0.1$ can result in considerable $|\Delta \delta|$ and $|\Delta \sigma|$ but relatively small $\left|\Delta \theta_{23}\right|$. In the particular case of $a_{1}=0, \operatorname{Re}\left(\epsilon_{3}\right)=0.1$ contributes $\left|\Delta \theta_{23}\right| \simeq 0.06$ (or 0.10), $|\Delta \delta| \simeq 0.21$ (or 0.01 ) and $|\Delta \sigma| \simeq 0.14$ (or 0.02 ) for $\mathrm{NH}$ (or $\mathrm{IH}$ ), while $\operatorname{Im}\left(\epsilon_{3}\right)=0.1$ contributes $\left|\Delta \theta_{23}\right| \simeq 0.03$ (or 0.00), $|\Delta \delta| \simeq 0.12$ (or 0.10) and $|\Delta \sigma| \simeq 0.05$ (or 0.00). To summarize, $\epsilon_{1}$ is unlikely to induce considerable mixing-parameter deviations, while $\operatorname{Re}\left(\epsilon_{3}\right)$ is likely. Inversely, a considerable $\Delta \theta_{23}$ can be ascribed to $\operatorname{Re}\left(\epsilon_{3}\right)$, while a considerable $\Delta \delta$ may result from any symmetry-breaking parameter.

\section{RGE triggered symmetry breaking}

A flavor symmetry (FS) such as the $\mu-\tau$ reflection symmetry under study is usually introduced at an energy scale $\Lambda_{\mathrm{FS}}$ much higher than the electroweak (EW) one $\Lambda_{\mathrm{EW}} \sim$ $10^{2} \mathrm{GeV}[12,13]$. Therefore, the RGE effects should be taken into account when the flavorsymmetry model is confronted with the low-energy experimental data [71]. During the evolution process, the significant difference between the Yukawa coupling of $\mu$ and that of $\tau$ may provide a unique source for symmetry breaking. This section is just devoted to such a specific breaking of the $\mu-\tau$ reflection symmetry. At the one-loop level, the energy dependence of $M_{\nu}$ is described by [72-76]

$$
16 \pi^{2} \frac{\mathrm{d} M_{\nu}}{\mathrm{d} t}=C\left(Y_{l}^{\dagger} Y_{l}\right)^{\mathrm{T}} M_{\nu}+C M_{\nu}\left(Y_{l}^{\dagger} Y_{l}\right)+\alpha M_{\nu}
$$

where $t \equiv \ln \left(\mu / \mu_{0}\right)$ with $\mu$ denoting the renormalization scale, $C$ and $\alpha$ read

$$
\begin{aligned}
& C=-\frac{3}{2}, \quad \alpha \simeq-3 g_{2}^{2}+6 y_{t}^{2}+\lambda, \quad \text { in the SM ; } \\
& C=1, \quad \alpha \simeq-\frac{6}{5} g_{1}^{2}-6 g_{2}^{2}+6 y_{t}^{2}, \quad \text { in the MSSM . }
\end{aligned}
$$

In the basis of $M_{l}$ being diagonal, the Yukawa coupling matrix for charged leptons is given by $Y_{l}=\operatorname{Diag}\left(y_{e}, y_{\mu}, y_{\tau}\right)$. Due to $y_{e} \ll y_{\mu} \ll y_{\tau}$, it is reasonable to neglect the contributions of $y_{e}$ and $y_{\mu}$. In eq. (4.1), the $\alpha$-term is flavor universal and only contributes an overall rescaling factor $I_{\alpha}$ for the neutrino mass matrix, while the other two terms are able to modify its structure. Given an $M_{\nu}\left(\Lambda_{\mathrm{FS}}\right)$ of the form given by eq. (2.4) at $\Lambda_{\mathrm{FS}}$, integration of eq. (4.1) enables us to derive the RGE-corrected neutrino mass matrix at $\Lambda_{\mathrm{EW}}[77,78]$

$$
M_{\nu}\left(\Lambda_{\mathrm{EW}}\right)=I_{\alpha} I_{\tau}^{\dagger} M_{\nu}\left(\Lambda_{\mathrm{FS}}\right) I_{\tau}^{*}=I_{\alpha}\left[M_{\nu}\left(\Lambda_{\mathrm{FS}}\right)-\Delta_{\tau}\left(\begin{array}{ccc}
0 & 0 & M_{e \tau} \\
0 & 0 & M_{\mu \tau} \\
M_{e \tau} & M_{\mu \tau} & 2 M_{\tau \tau}
\end{array}\right)\right]
$$




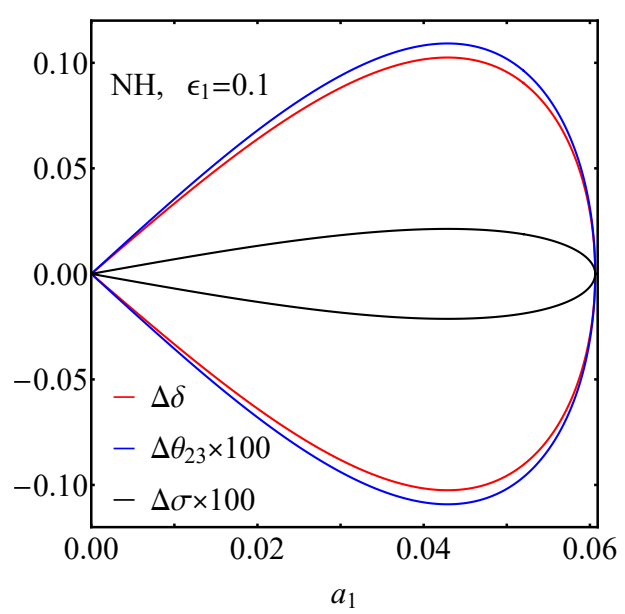

(a)

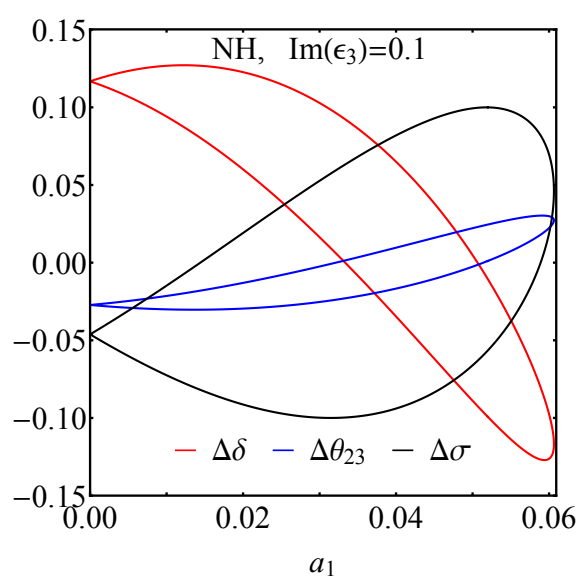

(e)

(c)

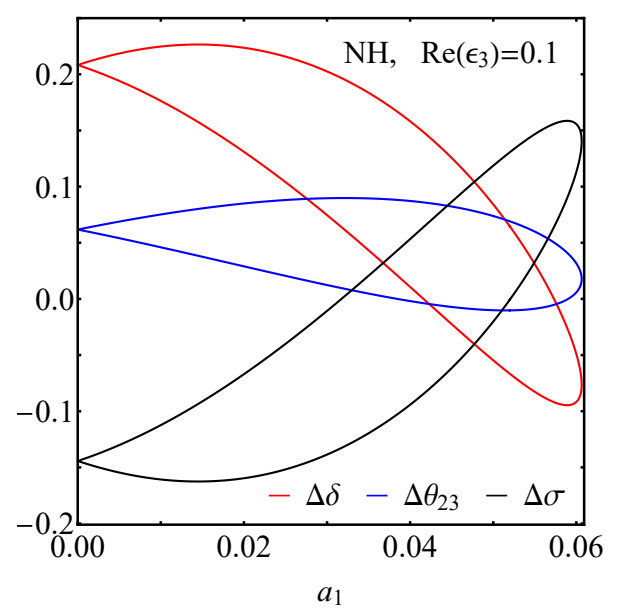

(e)

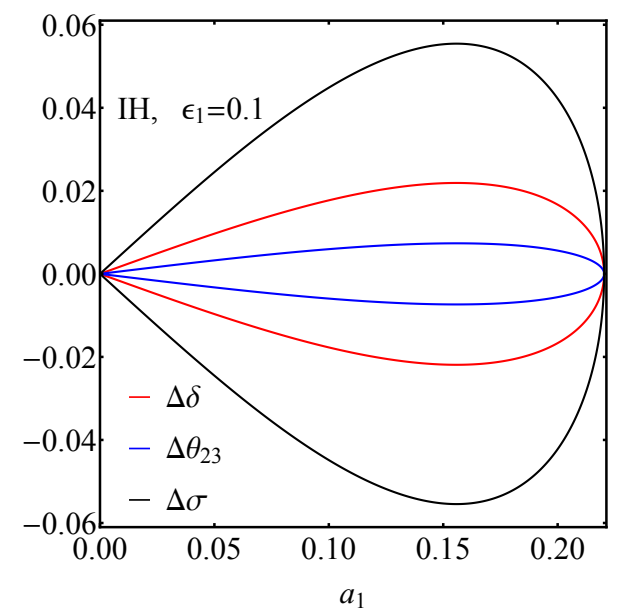

(b)

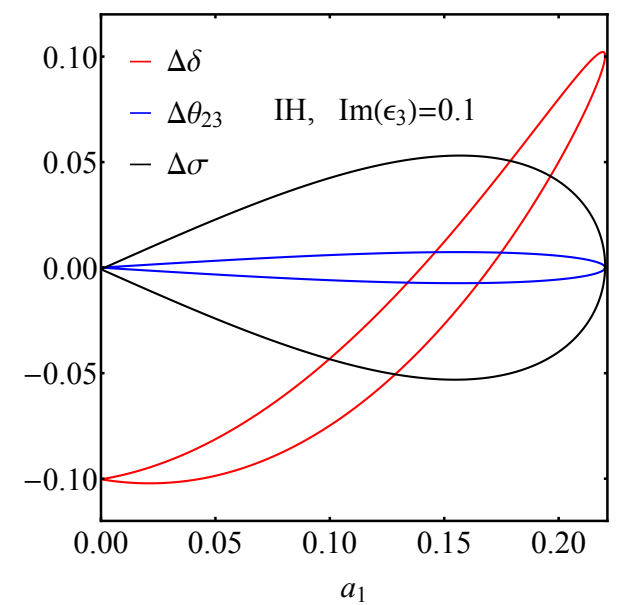

(d)

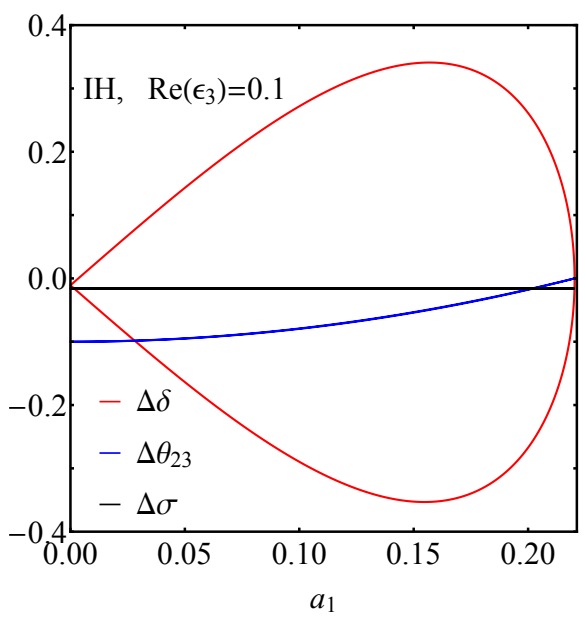

(f)

Figure 2. The $\Delta \theta_{23}, \Delta \delta$ and $\Delta \sigma$ (as functions of $a_{1}$ ) induced by $\epsilon_{1}=0.1, \operatorname{Re}\left(\epsilon_{3}\right)=0.1$ and $\operatorname{Im}\left(\epsilon_{3}\right)=0.1$ in the $\mathrm{NH}$ and $\mathrm{IH}$ cases. 
with $I_{\tau} \simeq \operatorname{Diag}\left(1,1,1-\Delta_{\tau}\right)$ and

$$
I_{\alpha}=\exp \left(-\frac{1}{16 \pi^{2}} \int_{\ln \Lambda_{\mathrm{EW}}}^{\ln \Lambda_{\mathrm{FS}}} \alpha \mathrm{dt}\right), \quad \Delta_{\tau}=\frac{C}{16 \pi^{2}} \int_{\ln \Lambda_{\mathrm{EW}}}^{\ln \Lambda_{\mathrm{FS}}} y_{\tau}^{2} \mathrm{dt} .
$$

Obviously, $\Delta_{\tau}$ measures the strength of symmetry breaking. Owing to the smallness of $y_{\tau} \simeq 0.01$ which gives $\Delta_{\tau} \simeq \mathcal{O}\left(10^{-5}\right)$, the RGE effect is negligible in the SM. But in the MSSM, $y_{\tau}^{2}=\left(1+\tan ^{2} \beta\right) m_{\tau}^{2} / v^{2}$ (with $v=174 \mathrm{GeV}$ being the Higgs VEV) can be greatly enhanced by a large $\tan \beta$. To be explicit, the value of $\Delta_{\tau}$ depends on $\tan \beta$ in a way as

$$
\Delta_{\tau} \simeq 0.042\left(\frac{\tan \beta}{50}\right)^{2}
$$

if we take $\Lambda_{\mathrm{FS}} \simeq 10^{13} \mathrm{GeV}$ as an example.

Following the same approach as in the previous section, one can obtain the following relations connecting the mixing-parameter deviations with $\Delta_{\tau}$

$$
\begin{aligned}
& m_{3} s_{13}^{2} \Delta \delta+\bar{m}_{2} s_{12}^{2} \Delta \sigma=\left(m_{1} c_{12}^{2}+\bar{m}_{2} s_{12}^{2}-m_{3} s_{13}^{2}\right)\left(-\Delta \phi_{1}\right), \\
& 2\left[\left(m_{1}-\bar{m}_{2}\right) c_{12} s_{12}+\mathrm{i}\left(m_{1} c_{12}^{2}+\bar{m}_{2} s_{12}^{2}+m_{3}\right) \bar{s}_{13}\right] \Delta \theta_{23} \\
& \quad-2\left(m_{1} c_{12}^{2}+\bar{m}_{2} s_{12}^{2}-m_{3}\right) \bar{s}_{13} \Delta \delta+4 \bar{m}_{2} s_{12}\left(\mathrm{i} c_{12}-s_{12} \bar{s}_{13}\right) \Delta \sigma \\
& \quad=\left[\mathrm{i}\left(m_{11}+m_{3}\right) \bar{s}_{13}-m_{12}\right]\left(\Delta_{\tau}-2 \mathrm{i} \Delta \phi_{1}-2 \mathrm{i} \Delta \phi_{2}\right), \\
& -2\left(m_{1} s_{12}^{2}+\bar{m}_{2} c_{12}^{2}-m_{3}\right) \Delta \theta_{23}+2 \bar{m}_{2} c_{12}\left(\mathrm{i} c_{12}-2 s_{12} \bar{s}_{13}\right) \Delta \sigma \\
& \quad+2\left[\left(m_{1}-\bar{m}_{2}\right) c_{12} s_{12}-\mathrm{i}\left(m_{1} c_{12}^{2}+\bar{m}_{2} s_{12}^{2}\right) \bar{s}_{13}\right] \bar{s}_{13} \Delta \delta \\
& \quad=\left[m_{1} s_{12}^{2}+\bar{m}_{2} c_{12}^{2}+m_{3}-2 \mathrm{i}\left(m_{1}-\bar{m}_{2}\right) c_{12} s_{12} \bar{s}_{13}\right]\left(\Delta_{\tau}-2 \mathrm{i} \Delta \phi_{2}\right),
\end{aligned}
$$

with $m_{1}=0$ (or $m_{3}=0$ ) in the $\mathrm{NH}$ (or $\left.\mathrm{IH}\right)$ case. Solving these equations gives

$$
\Delta \theta_{23}=+0.40 \Delta_{\tau}, \quad \Delta \delta=+0.66 \Delta_{\tau}, \quad \Delta \sigma=-0.02 \Delta_{\tau},
$$

in the $\mathrm{NH}$ case, or

$$
\Delta \theta_{23}=-0.52 \Delta_{\tau}, \quad \Delta \delta=-0.06 \Delta_{\tau}, \quad \Delta \sigma=-0.16 \Delta_{\tau},
$$

in the IH case. We subsequently show the dependence of these mixing-parameter deviations on the value of $\tan \beta$ (which varies from 10 to 50 ) in figure 3 . One can see that the mixing parameters are pretty stable against the RGE corrections. Even for $\tan \beta=50$, one merely has $\left|\Delta \theta_{23}\right| \simeq 0.017$ (or 0.021), $|\Delta \delta| \simeq 0.028$ (or 0.003) and $|\Delta \sigma| \simeq 0.001$ (or 0.007) in the $\mathrm{NH}$ (or IH) case.

\section{$5 \quad$ Leptogenesis and the symmetry breaking}

Besides accommodating the smallness of neutrino masses, the seesaw mechanism can also explain the observed baryon-antibaryon asymmetry of the Universe via the leptogenesis mechanism [79]: the CP-violating, lepton-number-violating and out-of-equilibrium decays of $N_{i}$ may generate a lepton-antilepton asymmetry which is eventually converted to the 


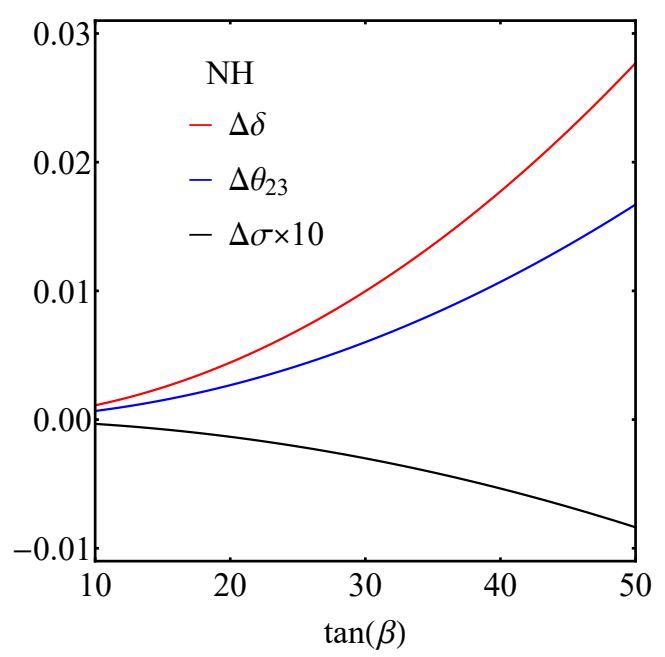

(a)

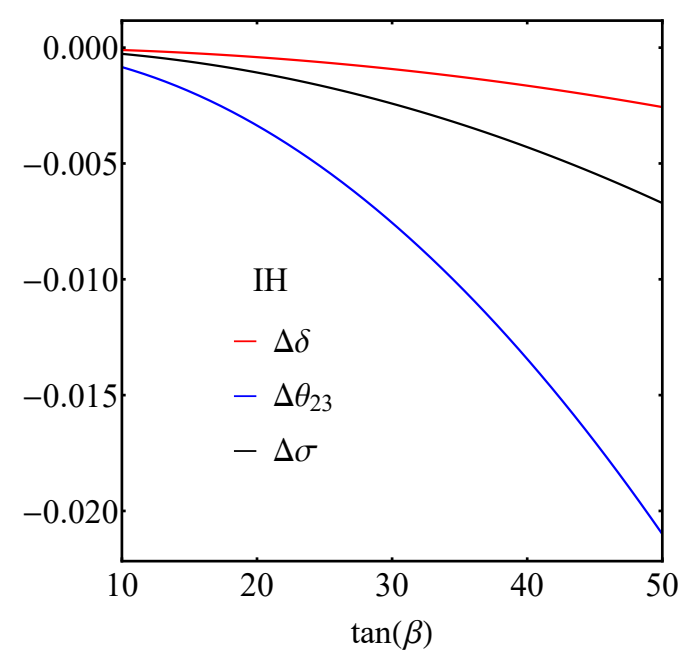

(b)

Figure 3. The RGE-induced $\Delta \theta_{23}, \Delta \delta$ and $\Delta \sigma$ against $\tan \beta$ in the MSSM.

baryon-antibaryon asymmetry through the sphaleron process [80]. The essential CP violation is provided by the complex Yukawa couplings $Y_{\mathrm{D}}=M_{\mathrm{D}} / v$ which couple $N_{i}$ with the left-handed neutrino fields, while the lepton-number violation originates from the Majorana mass terms of $N_{i}$. And the departure from thermal equilibrium can occur if $N_{i}$ decay in a rate smaller than the expansion rate of the Universe when the temperature $T$ drops to the mass scale of $N_{i}$. The produced amount of lepton-antilepton asymmetry is crucially dependent on the CP-violating asymmetries between the decays of $N_{i}$ and their CP conjugate processes. In the minimal seesaw under study, we assume $N_{1,2}$ to have a strong mass hierarchy $M_{1} \ll M_{2}$, in which case only the decay of $N_{1}$ is relevant for leptogenesis. The flavored CP-violating asymmetries $\varepsilon_{\alpha}$ are given by [81]

$$
\varepsilon_{\alpha} \simeq-\frac{3}{16 \pi\left(Y_{\mathrm{D}}^{\dagger} Y_{\mathrm{D}}\right)_{11}} \operatorname{Im}\left[\left(Y_{\mathrm{D}}^{\dagger} Y_{\mathrm{D}}\right)_{12}\left(Y_{\mathrm{D}}^{\dagger}\right)_{1 \alpha}\left(Y_{\mathrm{D}}\right)_{\alpha 2}\right] \frac{M_{1}}{M_{2}}
$$

for $\alpha=e, \mu, \tau$. A $Y_{\mathrm{D}}$ corresponding to the $M_{\mathrm{D}}$ in eq. (2.3) immediately yields [14]

$$
\varepsilon_{e}=0, \quad \varepsilon_{\mu}=-\varepsilon_{\tau},
$$

rendering the total $\mathrm{CP}$-violating asymmetry $\varepsilon=\varepsilon_{e}+\varepsilon_{\mu}+\varepsilon_{\tau}$ vanishing. However, a successful leptogenesis is possible when the $\mu-\tau$ reflection symmetry is broken [82] or (and) the flavor effects become relevant [83].

For the situation of $M_{1}>10^{12} \mathrm{GeV}$, the flavor effects are negligible. In order to achieve $\varepsilon \neq 0$, one has to break the $\mu$ - $\tau$ reflection symmetry. A $Y_{\mathrm{D}}^{\prime}$ corresponding to the $M_{\mathrm{D}}^{\prime}$ in eq. (3.3) (with $\epsilon_{2,4}=0$ as assumed before) gives

$$
\begin{aligned}
\varepsilon \simeq & \frac{3 M_{1}\left[a_{1} b_{1}+2 a_{2} b_{2} \cos \left(\phi_{a}-\phi_{b}\right)\right]}{8 v^{2}\left(a_{1}^{2}+2 a_{2}^{2}\right)} \\
& \times\left[a_{1} b_{1} \epsilon_{1}+2 a_{2} b_{2} \sin \left(\phi_{a}-\phi_{b}\right) \operatorname{Re}\left(\epsilon_{3}\right)+2 a_{2} b_{2} \cos \left(\phi_{a}-\phi_{b}\right) \operatorname{Im}\left(\epsilon_{3}\right)\right],
\end{aligned}
$$


which means that $\varepsilon$ is proportional to $M_{1}$ and a linear function of $\epsilon_{1}, \operatorname{Re}\left(\epsilon_{3}\right)$ and $\operatorname{Im}\left(\epsilon_{3}\right)$. The final baryon-to-entropy ratio can be written as [80]

$$
\mathrm{Y}_{\mathrm{B}} \equiv \frac{n_{\mathrm{B}}-n_{\overline{\mathrm{B}}}}{s} \simeq-\frac{12}{37} \kappa \frac{\varepsilon}{g_{*}} .
$$

Here $12 / 37$ is the efficiency factor of converting the lepton-antilepton asymmetry to the baryon-antibaryon asymmetry, whereas $g_{*}=106.75$ is the effective number of relativistic degrees of freedom at $T=M_{1}$ in the SM. In particular, $\kappa$ is the washout factor and can be parametrized as [80]

$$
\kappa \simeq(2 \pm 1) \times 10^{-2} \times\left(\frac{0.01 \mathrm{eV}}{\tilde{m}_{1}}\right)^{1.1 \pm 0.1}
$$

with $\tilde{m}_{1}=\left(Y_{\mathrm{D}}^{\dagger} Y_{\mathrm{D}}\right)_{11} v^{2} / M_{1}$. In the present epoch (for $s=7.04 n_{\gamma}$ ), the baryon-to-photon ratio is given by

$$
\eta \equiv \frac{n_{\mathrm{B}}-n_{\overline{\mathrm{B}}}}{n_{\gamma}} \simeq 7.04 \mathrm{Y}_{\mathrm{B}},
$$

which has an observed value of $(6.08 \pm 0.09) \times 10^{-10}$ [84]. To figure out what kind of $M_{1}$ and symmetry-breaking parameters may give rise to the observed baryon-antibaryon asymmetry, in figure 4 we present the $\eta$ (as functions of $a_{1}$ ) arising from some example values of them. The results show that a combination of $\left|M_{1} \epsilon_{1}\right| \simeq \mathcal{O}\left(10^{11}\right)$ (or $\left.\mathcal{O}\left(10^{13}\right)\right) \mathrm{GeV}$, $\left|M_{1} \operatorname{Im}\left(\epsilon_{3}\right)\right| \simeq \mathcal{O}\left(10^{10}\right)\left(\right.$ or $\left.\mathcal{O}\left(10^{13}\right)\right) \mathrm{GeV}$ or $\left|M_{1} \operatorname{Re}\left(\epsilon_{3}\right)\right| \simeq \mathcal{O}\left(10^{10}\right)\left(\right.$ or $\left.\mathcal{O}\left(10^{13}\right)\right) \mathrm{GeV}$ can give successful leptogenesis in the $\mathrm{NH}$ (or $\mathrm{IH})$ case. Clearly, it is much easier to gain the observed $\eta$ in the NH case than in the IH case. In particular, a $\left|\operatorname{Re}\left(\epsilon_{3}\right)\right|$ or $\left|\operatorname{Im}\left(\epsilon_{3}\right)\right| \simeq \mathcal{O}(0.01)$ (or smaller if $M_{1}$ takes a value larger than $10^{12} \mathrm{GeV}$ ) is sufficient for generating the observed $\eta$ in the NH case.

If $M_{1}$ turns out to be smaller than $10^{12} \mathrm{GeV}$, then the Yukawa interactions of charged leptons will enter in thermal equilibrium, making different lepton flavors distinguishable. In such a case, both the CP-violating asymmetries and washout factors associated with different flavors should be treated separately. Accordingly, the final baryon-to-entropy ratio can be rewritten as $[85,86]$

$$
\mathrm{Y}_{\mathrm{B}} \simeq-\frac{12}{37} \frac{K}{g_{*}},
$$

where the parameter $K$ is defined as

$$
\begin{aligned}
& K=\left(\epsilon_{e}+\epsilon_{\mu}\right) \kappa_{f}\left(\frac{417}{589} \tilde{m}_{e}+\frac{417}{589} \tilde{m}_{\mu}\right)+\epsilon_{\tau} \kappa_{f}\left(\frac{390}{589} \tilde{m}_{\tau}\right), \quad \text { if } 10^{9} \mathrm{GeV}<M_{1}<10^{12} \mathrm{GeV} \\
& K=\epsilon_{e} \kappa_{f}\left(\frac{151}{179} \tilde{m}_{e}\right)+\epsilon_{\mu} \kappa_{f}\left(\frac{344}{537} \tilde{m}_{\mu}\right)+\epsilon_{\tau} \kappa_{f}\left(\frac{344}{537} \tilde{m}_{\tau}\right), \quad \text { if } \quad M_{1}<10^{9} \mathrm{GeV}
\end{aligned}
$$

with

$$
\kappa_{f}\left(\tilde{m}_{\alpha}\right) \simeq\left[\left(\frac{\tilde{m}_{\alpha}}{2.1 \times 10^{-3} \mathrm{eV}}\right)^{-1}+\left(\frac{5 \times 10^{-4} \mathrm{eV}}{\tilde{m}_{\alpha}}\right)^{-1.16}\right]^{-1} \quad \text {, for } \tilde{m}_{\alpha} \equiv \frac{\left|\left(Y_{\mathrm{D}}\right)_{\alpha 1}\right|^{2} v^{2}}{M_{1}}
$$




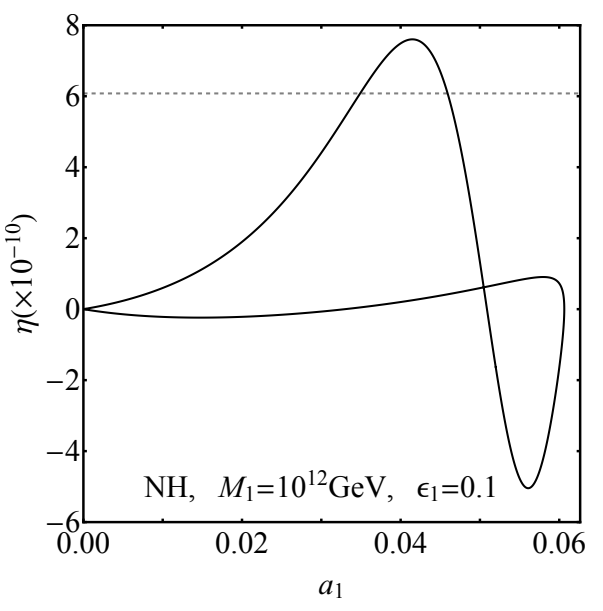

(a)

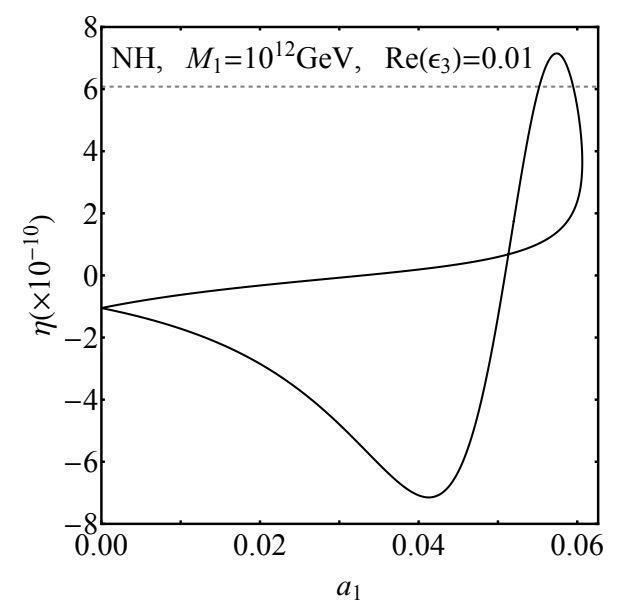

(c)

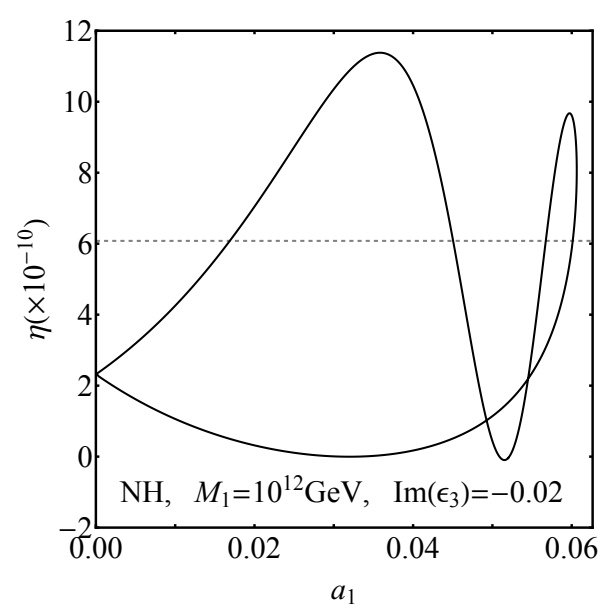

(e)

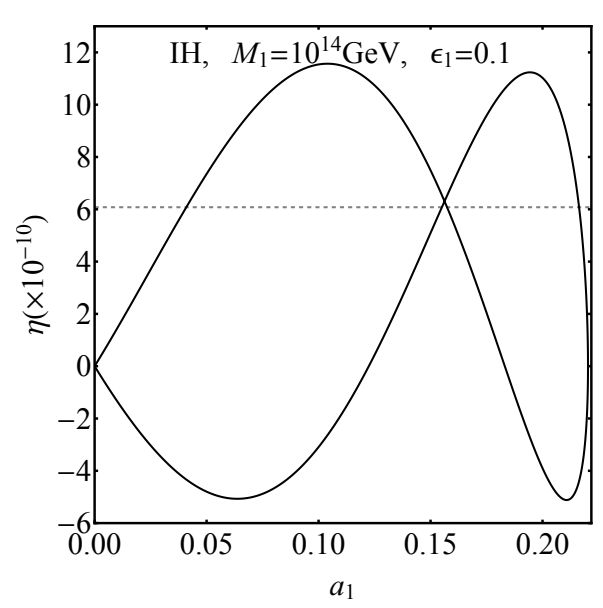

(b)

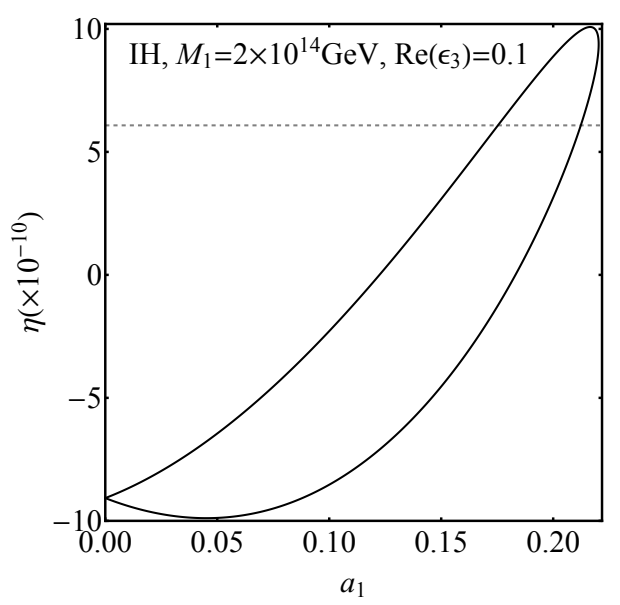

(d)

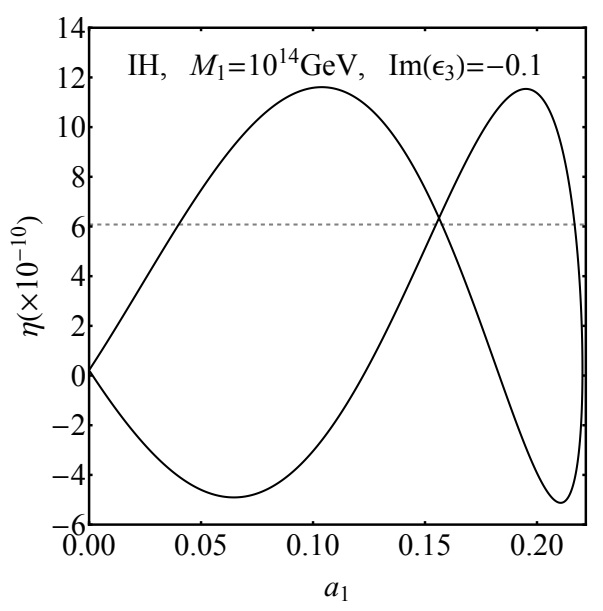

(f)

Figure 4. The $\eta$ (as functions of $a_{1}$ ) arising from some example values of $M_{1}$ and symmetrybreaking parameters for the situation of $M_{1}>10^{12} \mathrm{GeV}$. The gray dashed line stands for the observed value of $\eta$. 
For illustration, in figure 5 we show the $\eta$ (as functions of $a_{1}$ ) arising from some example values of $M_{1}$ and symmetry-breaking parameters for the situation of $10^{9} \mathrm{GeV}<M_{1}<$ $10^{12} \mathrm{GeV}$. For comparison, the contributions from pure flavor effects (without symmetrybreaking effects) are also shown. It is easy to see that the flavor effects are more significant than the symmetry-breaking effects in the NH case, while the contrary is the case in the $\mathrm{IH}$ case. In the $\mathrm{NH}$ case the flavor effects themselves are competent for generating the observed $\eta$. But in the IH case the symmetry-breaking effects have to be invoked and the symmetry-breaking parameters should take some values at least $\mathcal{O}(0.1)$.

In order to see whether there exists a choice of basic parameters which can lead to sizable $\Delta \delta, \Delta \theta_{23}$ and successful leptogenesis, in figures $6-7$ we present the resulting $\Delta \delta$ and $\Delta \theta_{23}$ from the parameter choices shown in figures $4-5$ that can lead to successful leptogenesis. From figure 6 one finds that for the situation of $M_{1}>10^{12} \mathrm{GeV}$ a sizable $\Delta \delta$ can be generated in association with a realistic $\eta$ from $\epsilon_{1}$ in the $\mathrm{NH}$ case (see the sub-figure labelled as (a)), $\operatorname{Re}\left(\epsilon_{3}\right)$ in the IH case (see the sub-figure labelled as (d)) or $\operatorname{Im}\left(\epsilon_{3}\right)$ in the IH case (see the sub-figure labelled as (f)), but a sizable $\Delta \theta_{23}$ has no chance to arise along with a successful leptogenesis. The results in figure 7 tell us that for the situation of $10^{9} \mathrm{GeV}<\mathrm{M}_{1}<10^{12} \mathrm{GeV}$ all the parameter choices except for that shown in the subfigure labelled as (b) may give rise to a sizable $\Delta \delta$, while only the parameter choice shown in the sub-figure labelled as (c) is capable of producing a sizable $\Delta \theta_{23}$.

\section{Summary}

The $\mu-\tau$ reflection symmetry is powerful in shaping the neutrino mixing: it leads to the interesting results $\theta_{23}=\pi / 4$ and $\delta= \pm \pi / 2$ (which are close to the current experimental results) as well as trivial Majorana phases. On the other hand, the minimal seesaw has strong predictive power in settling the neutrino mass spectrum: it enforces the condition of $m_{1}=0$ or $m_{3}=0$. In this paper, an attempt of implementing the $\mu-\tau$ reflection symmetry in the minimal seesaw has been made. Such a marriage results in $\sigma=\pi / 2$ (or 0 ) and thus $\left|M_{e e}\right| \simeq 0.37$ (or 4.86) eV in the $\mathrm{NH}$ (or $\mathrm{IH}$ ) case. Motivated by the preliminary experimental hints towards $\theta_{23} \neq \pi / 4$ and $\delta \neq-\pi / 2$, we particularly explore the possible symmetry breakings and their phenomenological consequences. Some parameters characterizing the breaking of $\mu-\tau$ refection symmetry are first defined and their implications for the mixing-parameter deviations then analyzed. It is found that $\epsilon_{1}$ is difficult to produce considerable mixing-parameter deviations, while $\operatorname{Re}\left(\epsilon_{3}\right)$ is relatively easy. Inversely, a considerable $\Delta \theta_{23}$ can be attributed to $\operatorname{Re}\left(\epsilon_{3}\right)$, while a considerable $\Delta \delta$ may arise from any symmetry-breaking parameter.

As a unique example, the symmetry breaking triggered by the RGE effects is studied. It turns out that the mixing parameters are rather stable against the RGE corrections. Even for $\tan \beta \simeq 50$ in the MSSM, the RGE-induced mixing-parameter deviations are only of $\mathcal{O}(0.01)$. Finally, the operation of leptogenesis in the framework under study is discussed. For the situation of $M_{1}>10^{12} \mathrm{GeV}$ where one has a vanishing $\varepsilon$, the $\mu$ $\tau$ reflection symmetry must be broken to make the leptogenesis mechanism work. For illustration, we give some example values of $M_{1}$ and symmetry-breaking parameters that 


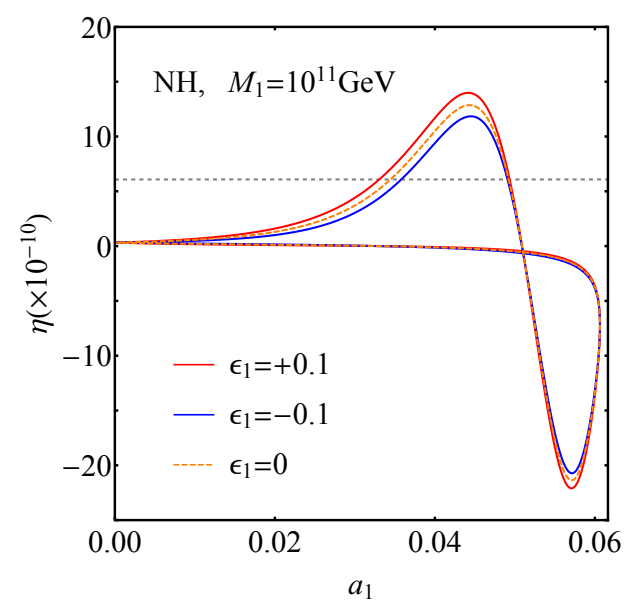

(a)

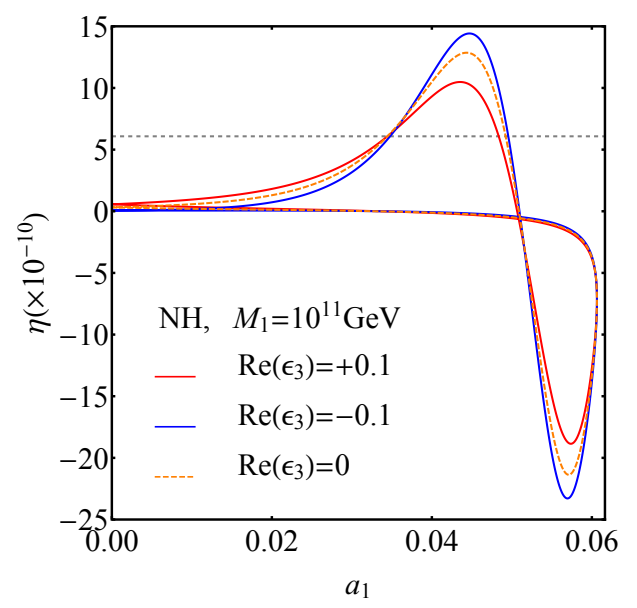

(c)

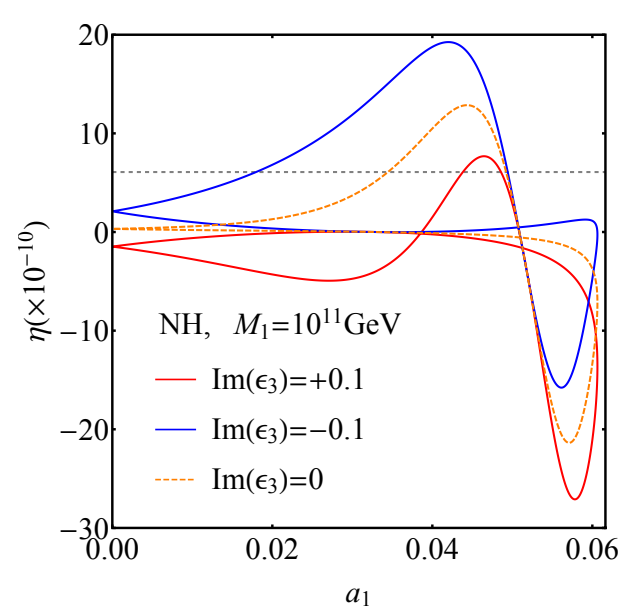

(e)

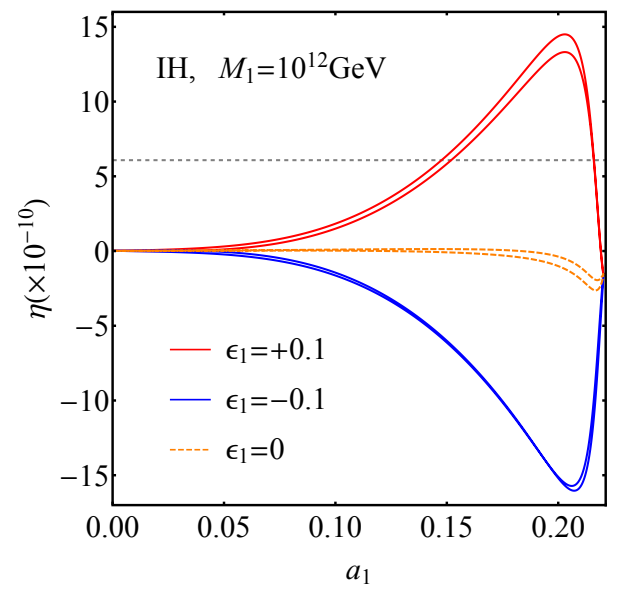

(b)

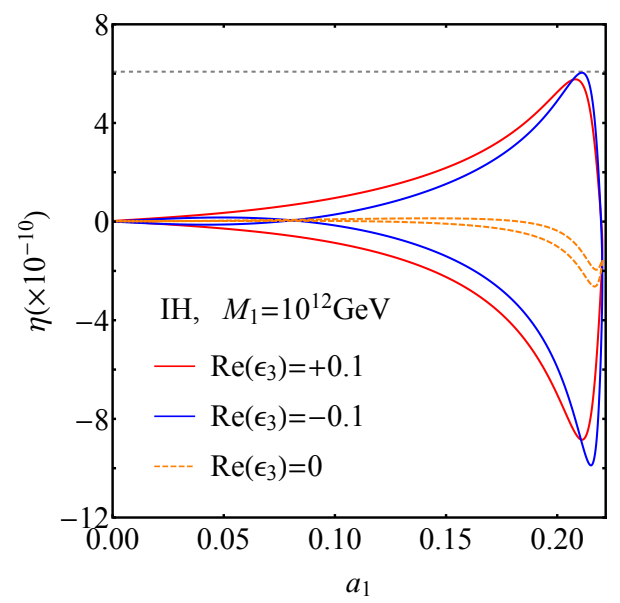

(d)

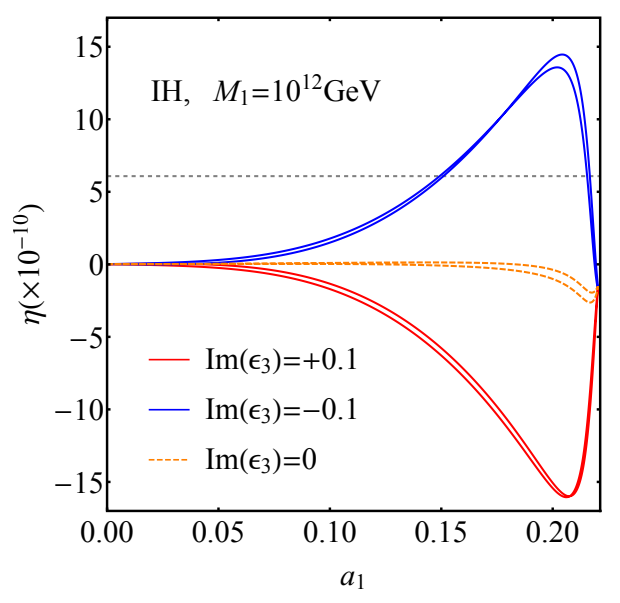

(f)

Figure 5. The $\eta$ (as functions of $a_{1}$ ) arising from some example values of $M_{1}$ and symmetrybreaking parameters for the situation of $10^{9} \mathrm{GeV}<\mathrm{M}_{1}<10^{12} \mathrm{GeV}$. The gray dashed line stands for the observed value of $\eta$. 


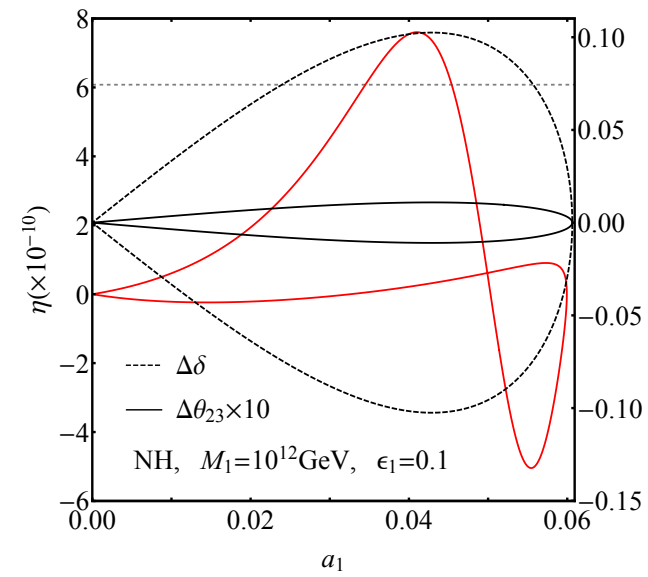

(a)

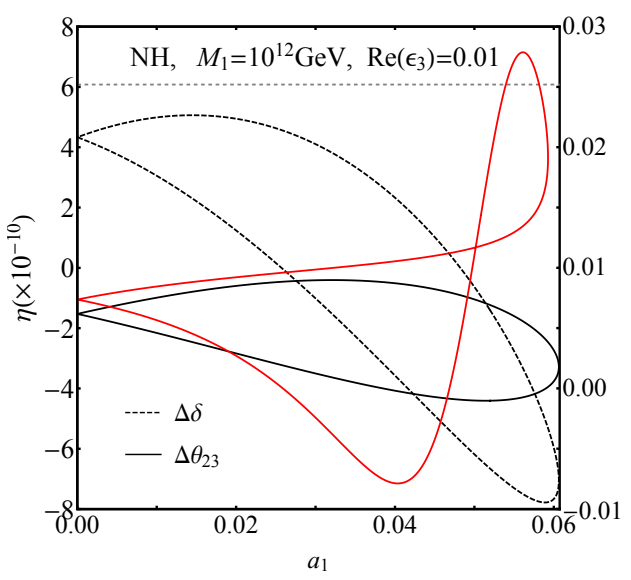

(c)

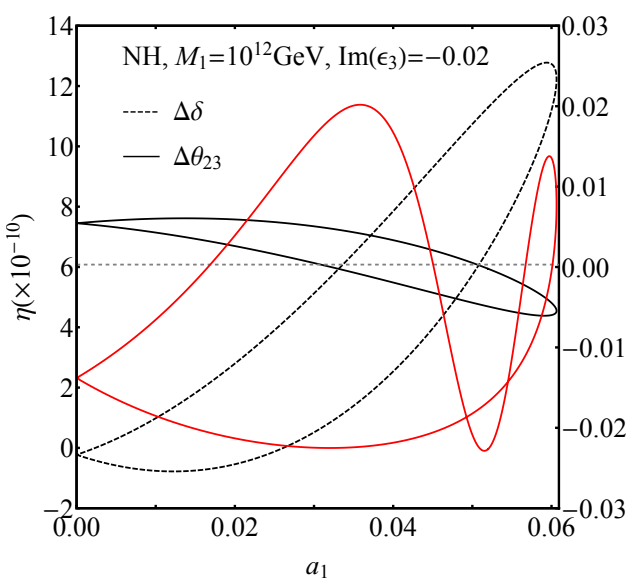

(e)

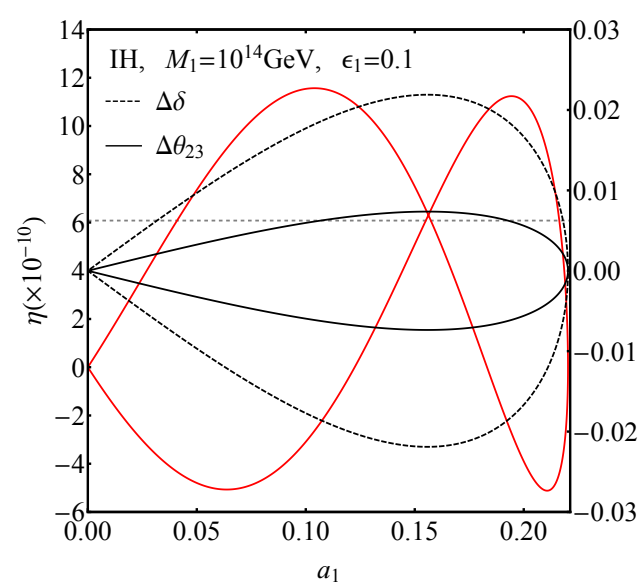

(b)

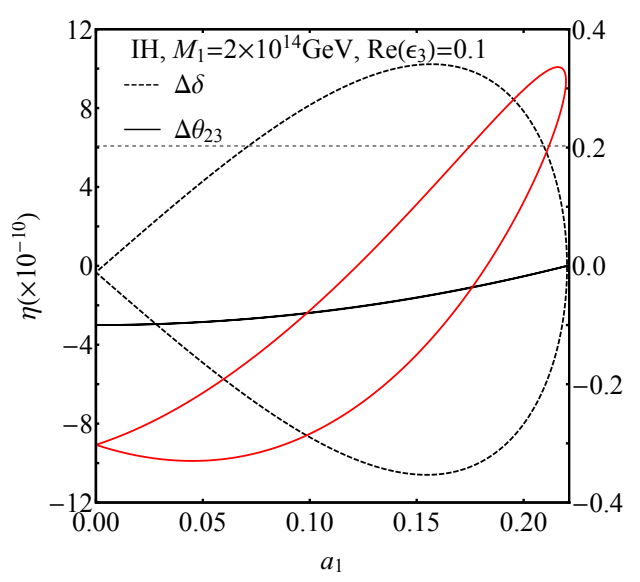

(d)

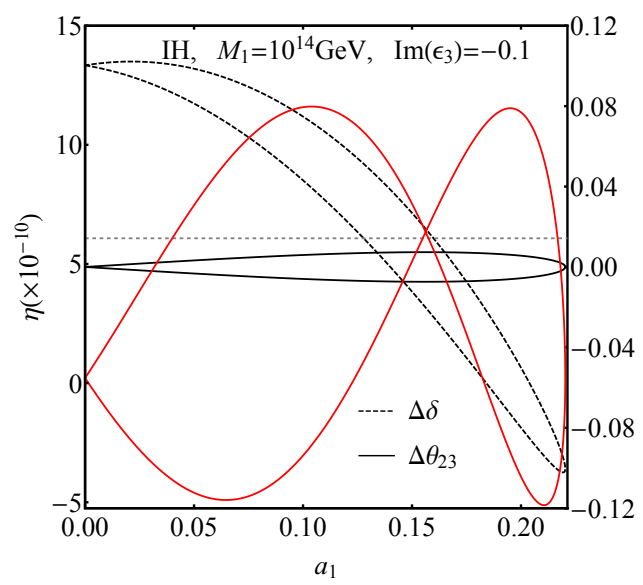

(f)

Figure 6. The resulting $\Delta \delta$ and $\Delta \theta_{23}$ (as functions of $a_{1}$ ) from the parameter choices shown in figure 4 that can lead to successful leptogenesis. The red line is used to denote the corresponding $\eta$. 


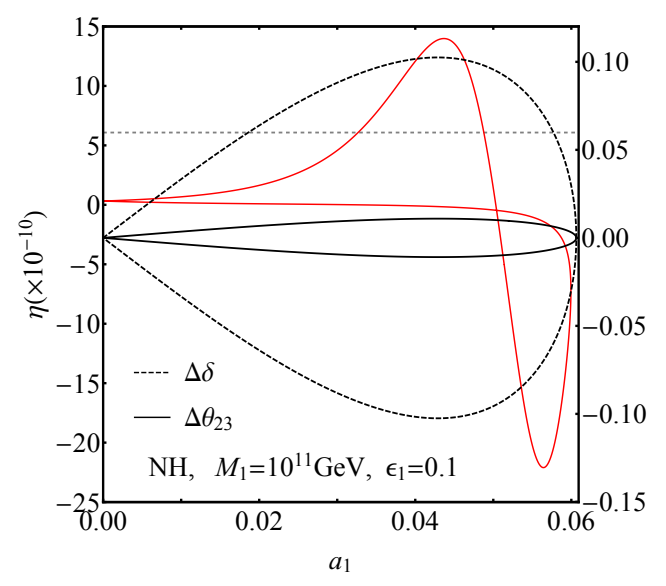

(a)

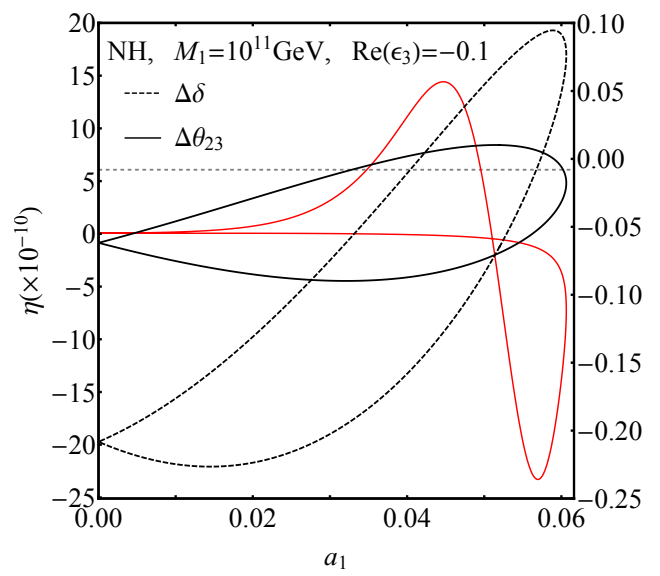

(c)

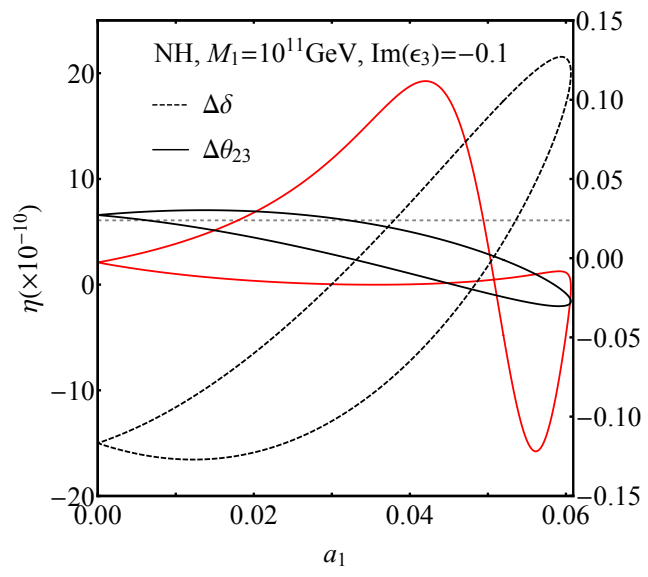

(e)

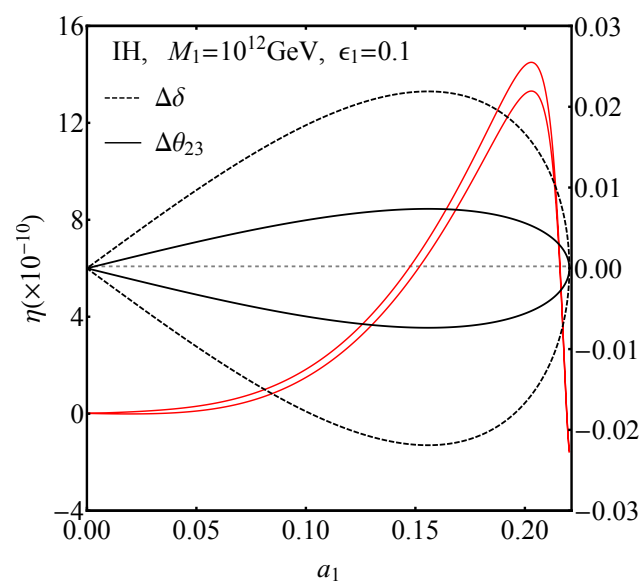

(b)

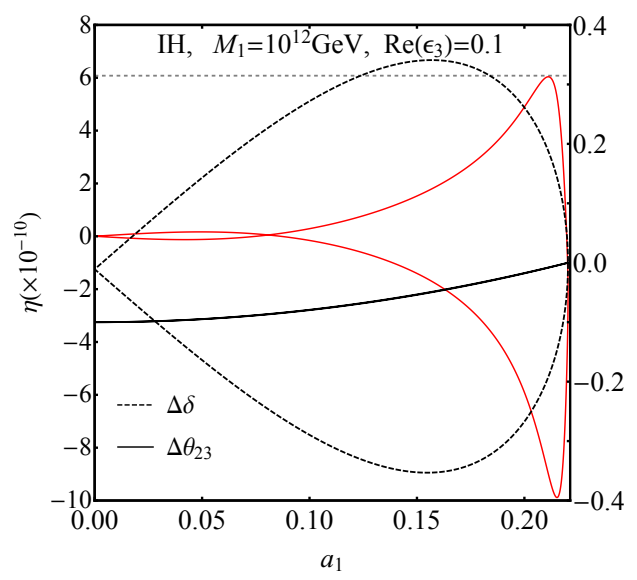

(d)

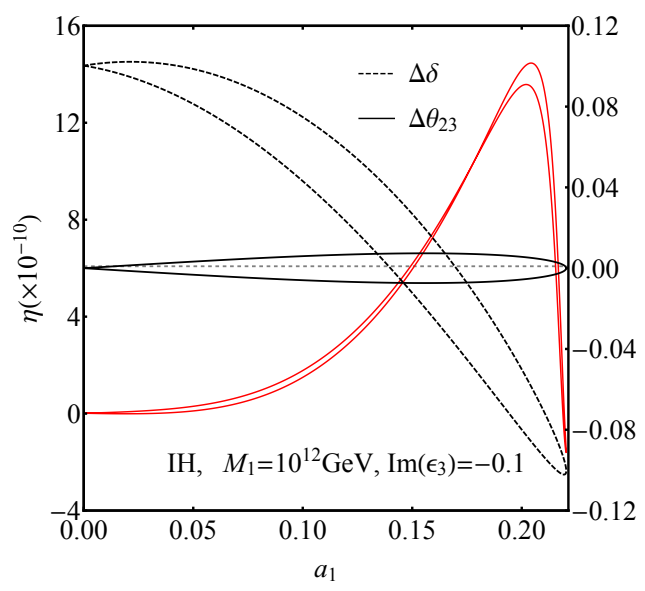

(f)

Figure 7. The resulting $\Delta \delta$ and $\Delta \theta_{23}$ (as functions of $a_{1}$ ) from the parameter choices shown in figure 5 that can lead to successful leptogenesis. The red line is used to denote the corresponding $\eta$. 
may give rise to the observed value of $\eta$. For the situation of $10^{9} \mathrm{GeV}<M_{1}<10^{12} \mathrm{GeV}$, it is possible that the flavor effects themselves (without symmetry-breaking effects) are sufficient for producing the observed $\eta$ in the $\mathrm{NH}$ case. But in the IH case, one has to turn to the symmetry-breaking effects for help. For both situations, it is easier to achieve a realistic value of $\eta$ in the $\mathrm{NH}$ case than in the $\mathrm{IH}$ case. Last but not least, we point out that the mixing-parameter deviations can be connected to the implementation of leptogenesis, considering that they may originate from the same symmetry breaking. The results show that a sizable $\Delta \delta$ can be generated in association with the observed $\eta$ in many cases, but a sizable $\Delta \theta_{23}$ can only arise along with a successful leptogenesis from $\operatorname{Re}\left(\epsilon_{3}\right)$ in the $\mathrm{NH}$ case for the situation of $10^{9} \mathrm{GeV}<\mathrm{M}_{1}<10^{12} \mathrm{GeV}$.

\section{Acknowledgments}

This work is supported in part by the National Natural Science Foundation of China under grant No. 11275088 (Z. C. L and C. X. Y) and grant No. 11605081 (Z. H. Z).

Open Access. This article is distributed under the terms of the Creative Commons Attribution License (CC-BY 4.0), which permits any use, distribution and reproduction in any medium, provided the original author(s) and source are credited.

\section{References}

[1] Particle Data Group collaboration, C. Patrignani et al., Review of particle physics, Chin. Phys. C 40 (2016) 100001 [inSPIRE].

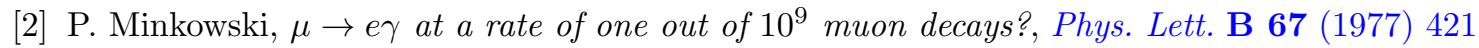
[INSPIRE].

[3] M. Gell-Mann, P. Ramond and R. Slansky, Complex spinors and unified theories, in Supergravity, P. van Nieuwenhuizen and D. Freedman eds., North-Holland, The Netherlands, (1979), pg. 315 [Conf. Proc. C 790927 (1979) 315] [arXiv: 1306.4669] [InSPIRE].

[4] T. Yanagida, Horizontal symmetry and masses of neutrinos, in Proceedings of the Workshop on the Unified Theory and the Baryon Number in the Universe, O. Sawada and A. Sugamoto eds., KEK report No. 79-18, Tsukuba Japan, (1979), pg. 95 [inSPIRE].

[5] R.N. Mohapatra and G. Senjanović, Neutrino mass and spontaneous parity violation, Phys. Rev. Lett. 44 (1980) 912 [INSPIRE].

[6] J. Schechter and J.W.F. Valle, Neutrino masses in $\mathrm{SU}(2) \times \mathrm{U}(1)$ theories, Phys. Rev. D 22 (1980) 2227 [InSPIRE].

[7] B. Pontecorvo, Neutrino experiments and the problem of conservation of leptonic charge, Sov. Phys. JETP 26 (1968) 984 [Zh. Eksp. Teor. Fiz. 53 (1967) 1717] [InSPIRE].

[8] Z. Maki, M. Nakagawa and S. Sakata, Remarks on the unified model of elementary particles, Prog. Theor. Phys. 28 (1962) 870 [InSPIRE].

[9] I. Esteban, M.C. Gonzalez-Garcia, M. Maltoni, I. Martinez-Soler and T. Schwetz, Updated fit to three neutrino mixing: exploring the accelerator-reactor complementarity, JHEP 01 (2017) 087 [arXiv: 1611.01514] [INSPIRE]. 
[10] Planck collaboration, P.A.R. Ade et al., Planck 2015 results. XIII. Cosmological parameters, Astron. Astrophys. 594 (2016) A13 [arXiv: 1502.01589] [INSPIRE].

[11] KATRIN collaboration, A. Osipowicz et al., KATRIN: a next generation tritium beta decay experiment with sub-eV sensitivity for the electron neutrino mass. Letter of intent, hep-ex/0109033 [INSPIRE].

[12] G. Altarelli and F. Feruglio, Discrete flavor symmetries and models of neutrino mixing, Rev. Mod. Phys. 82 (2010) 2701 [arXiv: 1002.0211] [INSPIRE].

[13] S.F. King and C. Luhn, Neutrino mass and mixing with discrete symmetry, Rept. Prog. Phys. 76 (2013) 056201 [arXiv: 1301.1340] [INSPIRE].

[14] P.F. Harrison and W.G. Scott, $\mu-\tau$ reflection symmetry in lepton mixing and neutrino oscillations, Phys. Lett. B 547 (2002) 219 [hep-ph/0210197] [INSPIRE].

[15] F. Feruglio, C. Hagedorn and R. Ziegler, Lepton mixing parameters from discrete and CP symmetries, JHEP 07 (2013) 027 [arXiv: 1211.5560] [INSPIRE].

[16] M. Holthausen, M. Lindner and M.A. Schmidt, CP and discrete flavour symmetries, JHEP 04 (2013) 122 [arXiv: 1211.6953] [INSPIRE].

[17] W. Grimus and L. Lavoura, A nonstandard CP transformation leading to maximal atmospheric neutrino mixing, Phys. Lett. B 579 (2004) 113 [hep-ph/0305309] [INSPIRE].

[18] T. Fukuyama and H. Nishiura, Mass matrix of Majorana neutrinos, hep-ph/9702253 [INSPIRE].

[19] E. Ma and M. Raidal, Neutrino mass, muon anomalous magnetic moment and lepton flavor nonconservation, Phys. Rev. Lett. 87 (2001) 011802 [Erratum ibid. 87 (2001) 159901] [hep-ph/0102255] [INSPIRE].

[20] C.S. Lam, A 2-3 symmetry in neutrino oscillations, Phys. Lett. B 507 (2001) 214 [hep-ph/0104116] [INSPIRE].

[21] K.R.S. Balaji, W. Grimus and T. Schwetz, The solar LMA neutrino oscillation solution in the Zee model, Phys. Lett. B 508 (2001) 301 [hep-ph/0104035] [INSPIRE].

[22] Z.-Z. Xing and Z.-H. Zhao, A review of $\mu-\tau$ flavor symmetry in neutrino physics, Rept. Prog. Phys. 79 (2016) 076201 [arXiv: 1512.04207] [INSPIRE].

[23] P.M. Ferreira, W. Grimus, L. Lavoura and P.O. Ludl, Maximal CP-violation in lepton mixing from a model with $\Delta(27)$ flavour symmetry, JHEP 09 (2012) 128 [arXiv:1206.7072] [INSPIRE].

[24] W. Grimus and L. Lavoura, $\mu-\tau$ interchange symmetry and lepton mixing, Fortsch. Phys. 61 (2013) 535 [arXiv:1207.1678] [INSPIRE].

[25] R.N. Mohapatra and C.C. Nishi, $S_{4}$ flavored CP symmetry for neutrinos, Phys. Rev. D 86 (2012) 073007 [arXiv: 1208.2875] [INSPIRE].

[26] Y.-L. Zhou, $\mu-\tau$ reflection symmetry and radiative corrections, arXiv:1409.8600 [INSPIRE].

[27] E. Ma, A. Natale and O. Popov, Neutrino mixing and CP phase correlations, Phys. Lett. B 746 (2015) 114 [arXiv: 1502.08023] [INSPIRE].

[28] E. Ma, Transformative $A_{4}$ mixing of neutrinos with CP-violation, Phys. Rev. D 92 (2015) 051301 [arXiv: 1504.02086] [INSPIRE].

[29] E. Ma, Neutrino mixing: $A_{4}$ variations, Phys. Lett. B 752 (2016) 198 [arXiv:1510.02501] [INSPIRE]. 
[30] A.S. Joshipura and K.M. Patel, Generalized $\mu-\tau$ symmetry and discrete subgroups of $O(3)$, Phys. Lett. B 749 (2015) 159 [arXiv:1507.01235] [InSPIRE].

[31] H.-J. He, W. Rodejohann and X.-J. Xu, Origin of constrained maximal CP-violation in flavor symmetry, Phys. Lett. B 751 (2015) 586 [arXiv:1507.03541] [INSPIRE].

[32] C.C. Nishi, New and trivial CP symmetry for extended $A_{4}$ flavor, Phys. Rev. D 93 (2016) 093009 [arXiv: 1601.00977] [INSPIRE].

[33] P.M. Ferreira, W. Grimus, D. Jurciukonis and L. Lavoura, Scotogenic model for co-bimaximal mixing, JHEP 07 (2016) 010 [arXiv: 1604.07777] [INSPIRE].

[34] A.S. Joshipura and N. Nath, Neutrino masses and mixing in $A_{5}$ with flavor antisymmetry, Phys. Rev. D 94 (2016) 036008 [arXiv: 1606.01697] [INSPIRE].

[35] P. Chen, G.-J. Ding, F. Gonzalez-Canales and J.W.F. Valle, Generalized $\mu-\tau$ reflection symmetry and leptonic CP-violation, Phys. Lett. B 753 (2016) 644 [arXiv:1512.01551] [INSPIRE].

[36] C.C. Nishi and B.L. Sánchez-Vega, $\mu-\tau$ reflection symmetry with a texture-zero, JHEP 01 (2017) 068 [arXiv: 1611.08282] [INSPIRE].

[37] Z.-H. Zhao, Breakings of the neutrino $\mu-\tau$ reflection symmetry, JHEP 09 (2017) 023 [arXiv: 1703.04984] [INSPIRE].

[38] W. Rodejohann and X.-J. Xu, Trimaximal $\mu-\tau$ reflection symmetry, Phys. Rev. D 96 (2017) 055039 [arXiv: 1705.02027] [INSPIRE].

[39] Z.-Z. Xing and J.-Y. Zhu, Neutrino mass ordering and $\mu-\tau$ reflection symmetry breaking, arXiv: 1707.03676 [INSPIRE].

[40] Z.-Z. Xing, D. Zhang and J.-Y. Zhu, The $\mu-\tau$ reflection symmetry of Dirac neutrinos and its breaking effect via quantum corrections, arXiv:1708.09144 [INSPIRE].

[41] A. Yu. Smirnov, Seesaw enhancement of lepton mixing, Phys. Rev. D 48 (1993) 3264 [hep-ph/9304205] [INSPIRE].

[42] S.F. King, Large mixing angle $M S W$ and atmospheric neutrinos from single right-handed neutrino dominance and U(1) family symmetry, Nucl. Phys. B 576 (2000) 85 [hep-ph/9912492] [INSPIRE].

[43] S.F. King, Constructing the large mixing angle MNS matrix in seesaw models with right-handed neutrino dominance, JHEP 09 (2002) 011 [hep-ph/0204360] [INSPIRE].

[44] S.F. King, Minimal predictive see-saw model with normal neutrino mass hierarchy, JHEP 07 (2013) 137 [arXiv:1304.6264] [INSPIRE].

[45] P.H. Frampton, S.L. Glashow and T. Yanagida, Cosmological sign of neutrino CP-violation, Phys. Lett. B 548 (2002) 119 [hep-ph/0208157] [INSPIRE].

[46] M. Raidal and A. Strumia, Predictions of the most minimal seesaw model, Phys. Lett. B 553 (2003) 72 [hep-ph/0210021] [INSPIRE].

[47] G.C. Branco, R. Gonzalez Felipe, F.R. Joaquim, I. Masina, M.N. Rebelo and C.A. Savoy, Minimal scenarios for leptogenesis and CP-violation, Phys. Rev. D 67 (2003) 073025 [hep-ph/0211001] [INSPIRE].

[48] V. Barger, D.A. Dicus, H.-J. He and T.-J. Li, Structure of cosmological CP-violation via neutrino seesaw, Phys. Lett. B 583 (2004) 173 [hep-ph/0310278] [InSPIRE].

[49] W.-L. Guo and Z.-Z. Xing, Calculable CP-violating phases in the minimal seesaw model of leptogenesis and neutrino mixing, Phys. Lett. B 583 (2004) 163 [hep-ph/0310326] [INSPIRE]. 
[50] J.-W. Mei and Z.-Z. Xing, Radiative corrections to neutrino mixing and CP-violation in the minimal seesaw model with leptogenesis, Phys. Rev. D 69 (2004) 073003 [hep-ph/0312167] [INSPIRE].

[51] A. Ibarra and G.G. Ross, Neutrino phenomenology: the case of two right-handed neutrinos, Phys. Lett. B 591 (2004) 285 [hep-ph/0312138] [INSPIRE].

[52] W.-L. Guo, Z.-Z. Xing and S. Zhou, Neutrino masses, lepton flavor mixing and leptogenesis in the minimal seesaw model, Int. J. Mod. Phys. E 16 (2007) 1 [hep-ph/0612033] [InSPIRE].

[53] H. Zhang and S. Zhou, The minimal seesaw model at the TeV scale, Phys. Lett. B 685 (2010) 297 [arXiv:0912.2661] [INSPIRE].

[54] K. Harigaya, M. Ibe and T.T. Yanagida, Seesaw mechanism with Occam's razor, Phys. Rev. D 86 (2012) 013002 [arXiv:1205.2198] [INSPIRE].

[55] J. Zhang and S. Zhou, A further study of the Frampton-Glashow-Yanagida model for neutrino masses, flavor mixing and baryon number asymmetry, JHEP 09 (2015) 065 [arXiv: 1505.04858] [INSPIRE].

[56] F. Björkeroth, F.J. de Anda, I. de Medeiros Varzielas and S.F. King, Leptogenesis in minimal predictive seesaw models, JHEP 10 (2015) 104 [arXiv:1505.05504] [INSPIRE].

[57] S.F. King, J. Zhang and S. Zhou, Renormalisation group corrections to the littlest seesaw model and maximal atmospheric mixing, JHEP 12 (2016) 023 [arXiv:1609.09402] [INSPIRE].

[58] T. Rink and K. Schmitz, Perturbed Yukawa textures in the minimal seesaw model, JHEP 03 (2017) 158 [arXiv : 1611.05857] [INSPIRE].

[59] T. Rink, K. Schmitz and T.T. Yanagida, Minimal seesaw model with a discrete heavy-neutrino exchange symmetry, arXiv:1612.08878 [INSPIRE].

[60] C.-C. Li and G.-J. Ding, Implications of residual CP symmetry for leptogenesis in a model with two right-handed neutrinos, Phys. Rev. D 96 (2017) 075005 [arXiv:1701.08508] [INSPIRE].

[61] G.-J. Ding, S.F. King and C.-C. Li, Golden littlest seesaw, arXiv:1705.05307 [InSPIRE].

[62] NOvA collaboration, P. Adamson et al., Measurement of the neutrino mixing angle $\theta_{23}$ in NOvA, Phys. Rev. Lett. 118 (2017) 151802 [arXiv:1701.05891] [INSPIRE].

[63] W. Rodejohann, Neutrino-less double beta decay and particle physics, Int. J. Mod. Phys. E 20 (2011) 1833 [arXiv:1106.1334] [INSPIRE].

[64] S.M. Bilenky and C. Giunti, Neutrinoless double-beta decay: a probe of physics beyond the Standard Model, Int. J. Mod. Phys. A 30 (2015) 1530001 [arXiv:1411.4791] [INSPIRE].

[65] S. Dell'Oro, S. Marcocci, M. Viel and F. Vissani, Neutrinoless double beta decay: 2015 review, Adv. High Energy Phys. 2016 (2016) 2162659 [arXiv:1601.07512] [INSPIRE].

[66] J.D. Vergados, H. Ejiri and F. Šimkovic, Neutrinoless double beta decay and neutrino mass, Int. J. Mod. Phys. E 25 (2016) 1630007 [arXiv:1612.02924] [INSPIRE].

[67] R.N. Mohapatra, $\theta_{13}$ as a probe of $\mu-\tau$ symmetry for leptons, JHEP 10 (2004) 027 [hep-ph/0408187] [INSPIRE].

[68] T. Kitabayashi and M. Yasuè, Maximal CP-violation in minimal seesaw model, Phys. Rev. D 94 (2016) 075020 [arXiv: 1605. 04402] [INSPIRE]. 
[69] W. Grimus, A.S. Joshipura, S. Kaneko, L. Lavoura, H. Sawanaka and M. Tanimoto, Non-vanishing $U_{e 3}$ and $\cos 2 \theta_{23}$ from a broken $Z_{2}$ symmetry, Nucl. Phys. B 713 (2005) 151 [hep-ph/0408123] [INSPIRE].

[70] S. Gupta, A.S. Joshipura and K.M. Patel, How good is $\mu-\tau$ symmetry after results on non-zero $\theta_{13}$ ?, JHEP 09 (2013) 035 [arXiv: 1301.7130] [INSPIRE].

[71] T. Ohlsson and S. Zhou, Renormalization group running of neutrino parameters, Nature Commun. 5 (2014) 5153 [arXiv:1311.3846] [INSPIRE].

[72] P.H. Chankowski and Z. Pluciennik, Renormalization group equations for seesaw neutrino masses, Phys. Lett. B 316 (1993) 312 [hep-ph/9306333] [INSPIRE].

[73] K.S. Babu, C.N. Leung and J.T. Pantaleone, Renormalization of the neutrino mass operator, Phys. Lett. B 319 (1993) 191 [hep-ph/9309223] [InSPIRE].

[74] S. Antusch, M. Drees, J. Kersten, M. Lindner and M. Ratz, Neutrino mass operator renormalization revisited, Phys. Lett. B 519 (2001) 238 [hep-ph/0108005] [INSPIRE].

[75] S. Antusch, M. Drees, J. Kersten, M. Lindner and M. Ratz, Neutrino mass operator renormalization in two Higgs doublet models and the MSSM, Phys. Lett. B 525 (2002) 130 [hep-ph/0110366] [INSPIRE].

[76] S. Antusch, J. Kersten, M. Lindner and M. Ratz, Running neutrino masses, mixings and CP phases: analytical results and phenomenological consequences, Nucl. Phys. B 674 (2003) 401 [hep-ph/0305273] [INSPIRE].

[77] J.R. Ellis and S. Lola, Can neutrinos be degenerate in mass?, Phys. Lett. B 458 (1999) 310 [hep-ph/9904279] [INSPIRE].

[78] P.H. Chankowski, W. Krolikowski and S. Pokorski, Fixed points in the evolution of neutrino mixings, Phys. Lett. B 473 (2000) 109 [hep-ph/9910231] [InSPIRE].

[79] M. Fukugita and T. Yanagida, Baryogenesis without grand unification, Phys. Lett. B 174 (1986) 45 [INSPIRE].

[80] W. Buchmüller, R.D. Peccei and T. Yanagida, Leptogenesis as the origin of matter, Ann. Rev. Nucl. Part. Sci. 55 (2005) 311 [hep-ph/0502169] [InSPIRE].

[81] Z.Z. Xing and S. Zhou, Neutrinos in particle physics, astronomy and cosmology, Springer-Verlag, Berlin Germany, (2011) [INSPIRE].

[82] Y.H. Ahn, S.K. Kang, C.S. Kim and T.P. Nguyen, Bridges of low energy observables with leptogenesis in $\mu-\tau$ reflection symmetry, arXiv:0811.1458 [INSPIRE].

[83] R.N. Mohapatra and C.C. Nishi, Implications of $\mu-\tau$ flavored CP symmetry of leptons, JHEP 08 (2015) 092 [arXiv: 1506.06788] [INSPIRE].

[84] WMAP collaboration, C.L. Bennett et al., Nine-year Wilkinson Microwave Anisotropy Probe (WMAP) observations: final maps and results, Astrophys. J. Suppl. 208 (2013) 20 [arXiv: 1212.5225] [INSPIRE].

[85] A. Abada, S. Davidson, F.-X. Josse-Michaux, M. Losada and A. Riotto, Flavor issues in leptogenesis, JCAP 04 (2006) 004 [hep-ph/0601083] [INSPIRE].

[86] E. Nardi, Y. Nir, E. Roulet and J. Racker, The importance of flavor in leptogenesis, JHEP 01 (2006) 164 [hep-ph/0601084] [INSPIRE]. 